\title{
Characterization of wind velocities in the upstream induction zone of a wind turbine using scanning continuous-wave lidars
}

Simley, Eric; Angelou, Nikolas; Mikkelsen, Torben Krogh; Sjöholm, Mikael; Mann, Jakob; Pao, Lucy Y.

Published in:

Journal of Renewable and Sustainable Energy

Link to article, DOI:

$10.1063 / 1.4940025$

Publication date:

2016

Document Version

Publisher's PDF, also known as Version of record

Link back to DTU Orbit

Citation (APA):

Simley, E., Angelou, N., Mikkelsen, T. K., Sjöholm, M., Mann, J., \& Pao, L. Y. (2016). Characterization of wind velocities in the upstream induction zone of a wind turbine using scanning continuous-wave lidars. Journal of Renewable and Sustainable Energy, 8(1), [013301]. https://doi.org/10.1063/1.4940025

\section{General rights}

Copyright and moral rights for the publications made accessible in the public portal are retained by the authors and/or other copyright owners and it is a condition of accessing publications that users recognise and abide by the legal requirements associated with these rights.

- Users may download and print one copy of any publication from the public portal for the purpose of private study or research.

- You may not further distribute the material or use it for any profit-making activity or commercial gain

- You may freely distribute the URL identifying the publication in the public portal 


\section{A J Journal of Renewable and Sustainable Energy}

Characterization of wind velocities in the upstream induction zone of a wind turbine using scanning continuous-wave lidars

Eric Simley, Nikolas Angelou, Torben Mikkelsen, Mikael Sjöholm, Jakob Mann, and Lucy Y. Pao

Citation: Journal of Renewable and Sustainable Energy 8, 013301 (2016); doi: 10.1063/1.4940025

View online: http://dx.doi.org/10.1063/1.4940025

View Table of Contents: http://scitation.aip.org/content/aip/journal/jrse/8/1?ver=pdfcov

Published by the AIP Publishing

\section{Articles you may be interested in}

Investigating wind turbine impacts on near-wake flow using profiling lidar data and large-eddy simulations with an actuator disk model

J. Renewable Sustainable Energy 7, 043143 (2015); 10.1063/1.4928873

New application of predictive direct torque control in permanent magnet synchronous generator-based wind turbine

J. Renewable Sustainable Energy 7, 023108 (2015); 10.1063/1.4915261

Streamwise development of the wind turbine boundary layer over a model wind turbine array

Phys. Fluids 25, 085108 (2013); 10.1063/1.4818451

Statistical analysis of kinetic energy entrainment in a model wind turbine array boundary layer

J. Renewable Sustainable Energy 4, 063105 (2012); 10.1063/1.4761921

Control strategies of doubly fed induction generator-based wind turbine system with new rotor current protection topology

J. Renewable Sustainable Energy 4, 043123 (2012); 10.1063/1.4748808

\section{AIP $\left.\right|_{\text {APL Photonics }}$}

APL Photonics is pleased to announce Benjamin Eggleton as its Editor-in-Chief

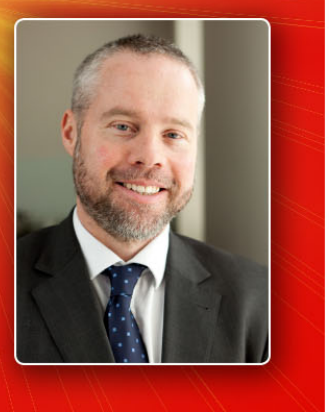




\title{
Characterization of wind velocities in the upstream induction zone of a wind turbine using scanning continuous-wave lidars
}

\author{
Eric Simley, ${ }^{1, a)}$ Nikolas Angelou, ${ }^{2}$ Torben Mikkelsen, ${ }^{2}$ Mikael Sjöholm, ${ }^{2}$ \\ Jakob Mann, ${ }^{2}$ and Lucy Y. Pao ${ }^{1}$ \\ ${ }^{1}$ Department of Electrical, Computer, and Energy Engineering, University of Colorado, \\ Boulder, Colorado 80309, USA \\ ${ }^{2}$ Department of Wind Energy, Technical University of Denmark, Ris $\phi$ Campus, \\ 4000 Roskilde, Denmark
}

(Received 19 March 2015; accepted 4 January 2016; published online 21 January 2016)

As a wind turbine generates power, induced velocities, lower than the freestream velocity, will be present upstream of the turbine due to perturbation of the flow by the rotor. In this study, the upstream induction zone of a $225 \mathrm{~kW}$ horizontal axis Vestas V27 wind turbine located at the Danish Technical University's Ris $\varnothing$ campus is investigated using a scanning Light Detection and Ranging (lidar) system. Three short-range continuous-wave "WindScanner" lidars are positioned in the field around the V27 turbine allowing detection of all three components of the wind velocity vectors within the induction zone. The time-averaged mean wind speeds at different locations in the upstream induction zone are measured by scanning a horizontal plane at hub height and a vertical plane centered at the middle of the rotor extending roughly 1.5 rotor diameters $(D)$ upstream of the rotor. Turbulence statistics in the induction zone are studied by more rapidly scanning along individual lines perpendicular to the rotor at different radial distances from the hub. The mean velocity measurements reveal that the longitudinal velocity reductions become greater closer to the rotor plane and closer to the center of the rotor. Velocity deficits of $1 \%-3 \%$ of the freestream value were observed $1 \mathrm{D}$ upstream of the rotor, increasing at the rotor plane to $7.4 \%$ near the edge of the rotor and $18 \%$ near the center of the rotor while the turbine was operating with a high estimated mechanical coefficient of power $\left(C_{P}\right)$ of 0.56 yielding an estimated axial induction factor of 0.25 . The velocity reductions relative to the freestream velocity become smaller when the turbine's coefficient of power decreases; for a low $C_{P}$ of 0.16 resulting in an estimated induction factor of 0.04 , the velocity deficits are $\sim 1 \%$ of the freestream value $1 D$ upstream of the rotor and only $6 \%$ at the rotor plane near the center of the rotor. Additionally, the mean radial wind speeds were found to increase close to the edge of the rotor disk indicating an expansion of the incoming flow around the rotor. Radial velocity magnitudes at the edge of the rotor disk of approximately $9 \%$ and $3 \%$ of the freestream longitudinal wind speed were measured for the abovementioned high and low $C_{P}$ values, respectively. Turbulence statistics, calculated using $2.5-\mathrm{min}$ time series, suggest that the standard deviation of the longitudinal wind component decreases close to the rotor, while the standard deviation of the radial wind component appears to increase. When the turbine was operating with a high $C_{P}$ of 0.54 resulting in an estimated induction factor of 0.22 , standard deviation decreases of up to $22 \%$ of the estimated freestream value and increases of up to

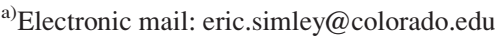


$46 \%$ were observed for the longitudinal and radial components, respectively, near the center of the rotor. (C) 2016 Author(s). All article content, except where otherwise noted, is licensed under a Creative Commons Attribution 3.0 Unported License. [http://dx.doi.org/10.1063/1.4940025]

\section{INTRODUCTION}

Due to the extraction of energy from the wind by a wind turbine, the velocities both upstream and downstream of the turbine are reduced compared to the freestream wind speed. A simple description of the velocity reductions is formed by treating a horizontal axis wind turbine rotor as an actuator disk and applying one-dimensional momentum theory (see Ref. 1, Chap. 3). As derived using the simple actuator disk model in steady flow, ${ }^{1}$ a turbine's power coefficient $C_{P}$, describing the fraction of the available power in the wind that is captured by the rotor, is a function of the axial induction factor $a$, where $a$ is defined as the fraction of the freestream wind speed $U_{\infty}$ that the wind speed is reduced by at the rotor disk

$$
a=\frac{U_{\infty}-U_{d i s k}}{U_{\infty}}
$$

where $U_{\text {disk }}$ is the wind speed at the rotor disk. Specifically, using one-dimensional momentum theory, the coefficient of power $C_{P}$ can be expressed as

$$
C_{P}=4 a(1-a)^{2}
$$

where $C_{P}$ is defined as

$$
C_{P}=\frac{P}{\frac{1}{2} \rho A U_{\infty}^{3}},
$$

where $P$ is the power produced by the rotor, $\rho$ is the air density, and $A$ is the rotor area. ${ }^{1}$ The well-known optimal induction factor that maximizes power capture is $a=1 / 3$. The region upstream of the turbine where the freestream velocity is gradually reduced to its value at the rotor disk $U_{\text {disk }}$ is referred to as the "upstream induction zone" or simply the "induction zone." Furthermore, due to the reduction in longitudinal wind speed (aligned with the mean wind direction) in the induction zone, application of conservation of mass using one-dimensional momentum theory indicates that a streamtube passing around the rotor disk expands as the distance upstream of the rotor decreases. ${ }^{1}$ Thus, the mean radial wind speeds (perpendicular to the longitudinal direction, oriented away from the rotor center) will be non-zero close to the rotor to produce this expansion.

A more detailed method for describing the velocities in a wind turbine's wake as well as the induced velocities in the induction zone relies on vortex theory. As described by Segalini and Alfredsson ${ }^{2}$ and Sarmast et al., ${ }^{3}$ the flow field around a turbine rotor can be approximated by modeling the blades as vortex lines with constant circulation and modeling the root vortex and blade tip vortices that are shed from the rotor. In this approach, which resolves the individual rotating blades rather than treating the rotor as an actuator disk, the wind speed deficits in the induction zone can be determined using the distribution of induced velocities produced by the tip vortices. The non-zero radial velocities near the rotor, which are predicted by the streamtube expansion using actuator disk theory, are more realistically explained as being induced by the tip vortices shed by the rotating blades.

As discussed by Segalini and Alfredsson ${ }^{2}$ and presented by Medici et al., ${ }^{4}$ the induced velocities at all distances upstream and downstream of the rotor along the "symmetry axis," which passes through the center of the rotor in the longitudinal direction, can be analytically determined using a simplified vortex model consisting of a cylindrical vortex sheet of helical 
tip vortices trailing behind the rotor. As provided in Eq. (1) by Medici et al., ${ }^{4}$ the wind speeds along the symmetry axis normalized by the freestream wind speed are given by

$$
\frac{U}{U_{\infty}}=1-a\left(1+\frac{2 x}{D}\left(1+\left(\frac{2 x}{D}\right)^{2}\right)^{-\frac{1}{2}}\right),
$$

where $x$ represents the distance from the rotor, with $x<0$ upstream of the rotor, and $D$ is the rotor diameter. Using this vortex sheet theory formula, the wind speeds along the symmetry axis are shown in Fig. 1(a) expressed in terms of $a$ and plotted as a function of the distance from the rotor in units of rotor diameters $D$. In addition to the velocity reductions in the induction zone, flow expansion around the rotor will occur, as explained by the streamtube expansion resulting from one-dimensional momentum theory and more realistically by the induced radial velocities produced by blade tip vortices. Figure 1(b) contains an illustration of this flow expansion using streamlines of the mean wind velocities around a rotor produced using the National Renewable Energy Laboratory (NREL)'s SOWFA computational fluid dynamics (CFD) tool with turbulent inflow using the NREL $5 \mathrm{MW}$ reference turbine model with an axial induction factor of $0.175 .^{5}$

The more severe downstream velocity deficits in a turbine's wake are of interest because turbines located in the wake of an upstream turbine can produce significantly less power than they would if they encountered undisturbed flow. But the velocity deficits in the upstream induction zone are of interest in certain wind energy applications as well. For example, velocity reductions in the induction zone can cause a bias in power curve measurements. ${ }^{6}$ If a turbine's power curve is calculated using reduced reference wind speed measurements in the induction zone, then the power curve will be overestimated. For this reason, the International Electrotechnical Commission recommends that for power curve calculations, reference measurements should be acquired at least 2 rotor diameters upstream of the turbine, where velocity deficits are estimated to be approximately $1 \%$ of the freestream wind speed. ${ }^{7}$ Additionally, preview-based control strategies relying on measurements of the approaching wind can be affected when the preview measurements occur in the induction zone..$^{5}$ In addition to the impact of using measurements of reduced wind speeds as control inputs, the non-zero radial wind

(a)

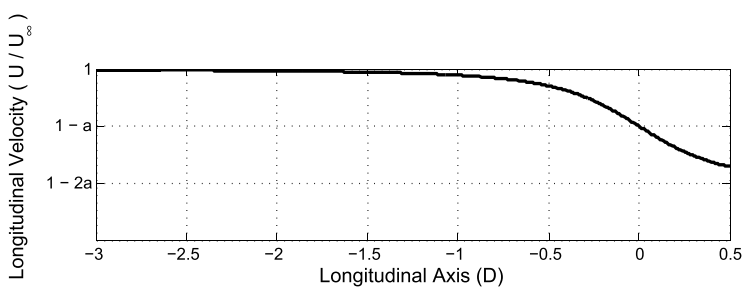

(b)

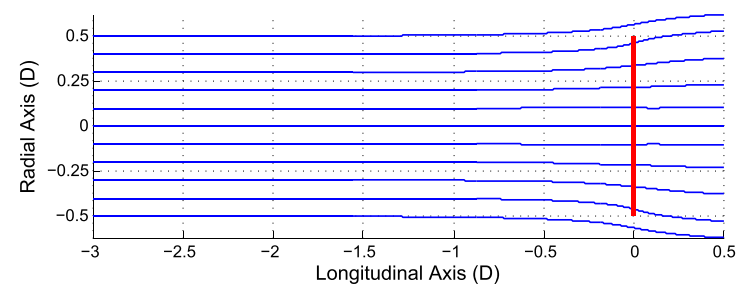

FIG. 1. (a) Example of mean longitudinal velocities along the axis perpendicular to the rotor center expressed in terms of freestream mean wind speed $U_{\infty}$, and axial induction factor $a$. Velocities calculated assuming an actuator disk in steady flow using the formula in Eq. (4), based on vortex sheet theory, presented by Medici et al. ${ }^{4}$ (b) Example of streamlines of the mean wind speeds in a wind turbine's induction zone. The radial and longitudinal axes are expressed in terms of the rotor diameter $D$ where negative longitudinal values are upstream of the turbine. Data obtained from a computational fluid dynamics simulation using the NREL $5 \mathrm{MW}$ reference turbine model with an axial induction factor of approximately $0.175 .^{5}$ 
speeds caused by the flow expansion can affect preview measurement quality. Assuming it is desirable to measure the wind that reaches the outer region of the rotor where more power is produced, the preview measurements should be made at a slightly smaller radial distance from the rotor center to account for the inflow expansion.

Several approaches have been previously used to investigate the induction zone. As discussed earlier, analytic vortex models have been developed to compute the three-dimensional mean flow field in the induction zone and wake., ${ }^{2,3}$ In an earlier work by Modarresi and Kirchhoff, ${ }^{8}$ an analytic model of the velocity field and streamlines upstream of a wind turbine rotor, treated as a porous disk, in steady flow is presented. Particle image velocimetry (PIV) and hot-wire anemometry were used by Medici et al. ${ }^{4}$ to measure the wind velocities upstream of rotating model turbines in a wind tunnel. High resolution longitudinal and radial velocities in the near upstream region of the $18 \mathrm{~cm}$ diameter rotor are provided from the PIV analysis. Hot-wire anemometry revealed that velocity reductions between $2 \%$ and $5 \%$ of the freestream wind speed were present two rotor diameters upstream of the turbine, while velocity deficits of less than $1 \%$ were detected $3 \mathrm{D}$ upstream of the turbine. Medici et al. ${ }^{4}$ also presented a CFD investigation of the induction zone, finding that the numerical simulations predict lower wind speed deficits in the induction zone than exhibited by the experimental results. At $2 D$ upstream of the turbine, velocity deficits of only $\sim 1 \%$ are produced using CFD. Recently, Light Detection and Ranging (lidar) remote sensing has been used to measure the induction zone on full-scale wind turbines. Slinger et al. ${ }^{9}$ verified the reduction in wind speed up to $1.5 \mathrm{D}$ upstream of the rotor by measuring at several upstream distances using a circularly scanning lidar located on the nacelle. Different power curves were measured using reference lidar measurements at five distances between $0.14 D$ and $2.5 \mathrm{D}$ upstream of the turbine. A nacelle lidar was also employed by Asimakopoulos et al. ${ }^{10}$ to determine how far upstream of the turbine the induction zone extends, using forward-staring measurements as well as a horizontal arc scan at hub height. The authors conclude that at the upstream distance of $2.5 \mathrm{D}$, where power curve measurements are conventionally performed, velocity reductions between $1 \%$ and $3 \%$ of the freestream velocity are still detectable.

In this study, three scanning ground-based lidars, the short-range "WindScanner" lidars, are used to measure the induction zone upstream of a $225 \mathrm{~kW}$ Vestas V27 wind turbine at the Danish Technical University (DTU) Wind Energy department's Risø campus. Whereas the aforementioned lidar-based induction zone studies relied on a single nacelle-mounted lidar to provide line-of-sight velocity measurements, the three ground-based lidars are used to solve for the three wind components, namely, the longitudinal, radial, and tangential (aligned with the rotational direction of the rotor) components. Previous three-beam lidar measurement campaigns are discussed by Mann et al. ${ }^{11}$ and Carbajo Fuertes et al. ${ }^{12}$ in which the lidars are used to determine 3D turbulence statistics near sonic anemometers, so that the lidar accuracy can be analyzed. However, unlike the work presented in this study, these investigations were performed using fixed lidar measurement locations. Earlier scanning lidar investigations include Iungo and Portè-Agel, ${ }^{13}$ in which two synchronized ground-based lidars are used to scan the wake behind a $2 \mathrm{MW}$ wind turbine, allowing the longitudinal and vertical velocities to be determined. Sjöholm et al., ${ }^{14}$ used the same WindScanner lidar system employed in this work to measure two-dimensional flow fields caused by helicopter downwash. Finally, a demonstration of three scanning long-range pulsed lidars synchronized in space and time measuring the flow near sonic anemometers at several different heights on a meteorological (met) tower is presented in Vasiljevic. ${ }^{15}$

The three synchronized WindScanner lidars are used in this work to determine the timeaveraged mean velocities as a function of longitudinal and radial position in the induction zone by scanning a horizontal plane at hub height on one side of the nacelle extending roughly 1.5 $D$ upstream of the rotor for various wind speeds resulting in a wide range of induction factors a. A similar scan is employed to measure the time-averaged mean velocities in a vertical plane extending from the bottom to the top of the rotor up to $1.5 \mathrm{D}$ upstream of the turbine. Therefore, while much of the focus of previous induction zone investigations was on finding the velocity deficits that are present far upstream of the turbine, this study focuses on the near 
region of the induction zone. Finally, the impact of the induction zone on turbulence statistics is analyzed by rapidly scanning along lines perpendicular to the rotor plane at different radial positions and forming velocity time series at regular intervals up to $1 D$ upstream of the rotor.

The remainder of this paper is organized as follows. Section II describes the experimental setup for the induction zone measurements. A description of the Vestas V27 wind turbine is included in Section II A. Section II B contains information about the WindScanner lidars used for the measurements, Section IIC summarizes the lidar data processing procedures, and Section IID discusses the different scan patterns employed. Finally, Section IIE contains a discussion of the potential lidar measurement error sources. The resulting measurements of 10-min time-averaged mean longitudinal, radial, and tangential velocities as a function of longitudinal and radial position in the induction zone are provided in Section IV for a variety of mean freestream wind speeds. Section IV A contains the results of the horizontal plane scans and Section IV B illustrates the results of a vertical plane scan. Turbulence statistics in the induction zone are presented in Section V. Finally, Section VI concludes the paper with a brief discussion of the experimental methods and results.

\section{EXPERIMENTAL METHODS}

The induction zone measurements are performed using the $27 \mathrm{~m}$ rotor diameter, $225 \mathrm{~kW}$ Vestas V27 wind turbine at DTU Wind Energy's Ris $\emptyset$ campus. As shown in Fig. 2, the turbine is located on relatively flat agricultural land approximately $225 \mathrm{~m}$ to the east of Roskilde fjord. The terrain is gently sloping up from the shore of the fjord and a met mast used for reference measurements is located approximately $73 \mathrm{~m}(2.7 \mathrm{D})$ away from the turbine at a heading of $283^{\circ}$. The positions of the scanning lidars around the turbine are chosen to produce measurements of the induction zone up to $\sim 1.5 \mathrm{D}$ upstream of the rotor for wind directions from $221^{\circ}$
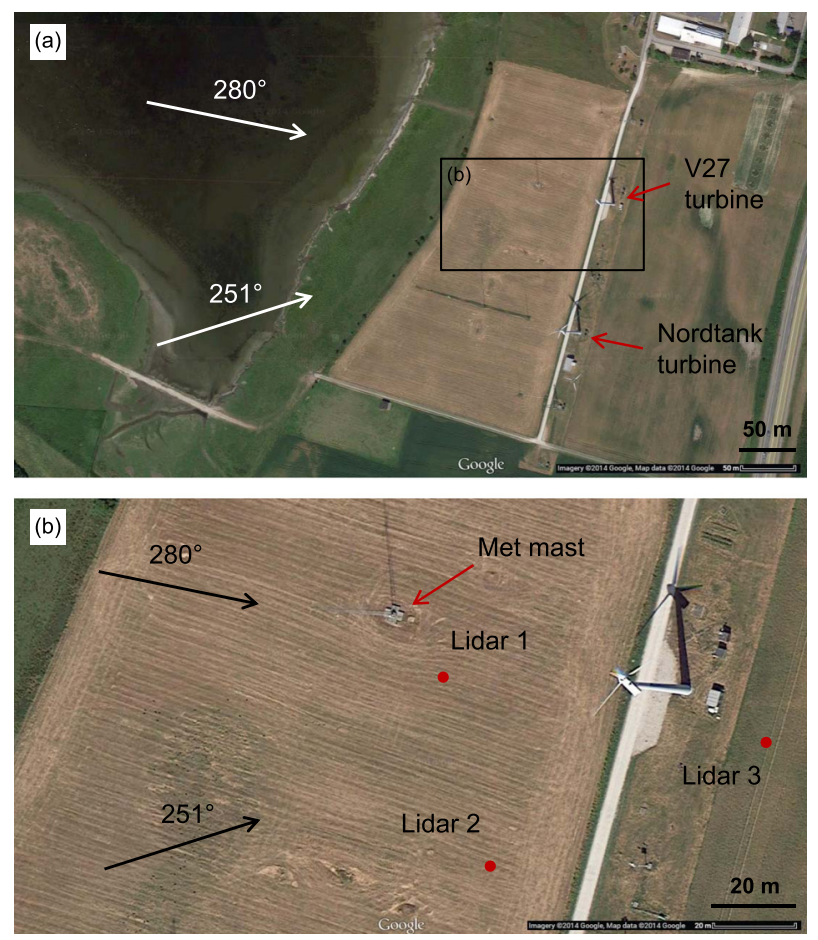

FIG. 2. Location of measurement campaign at the Danish Technical University's Ris $\varnothing$ campus. (a) Map of the terrain surrounding the Vestas V27 turbine used for the inflow measurements. Inflow measurements were acquired for intended wind directions between $251^{\circ}$ and $280^{\circ}$. (b) Close-up of the region near the V27 turbine where the experiment took place (indicated by the rectangular region in (a)). The locations of the three WindScanner lidars are indicated by the red points and are chosen to provide the highest inflow scan quality for a wind direction of $251^{\circ}$. Map imagery (C) 2014 Google, Map data (C) 2014 Google. 
to $281^{\circ}$, with an ideal wind direction of $251^{\circ}$, indicated in Fig. 2 . To improve the quality of the lidar scans, as will be explained in Section II B, the lidar positions are chosen to measure only the left side of the rotor when looking upstream.

\section{A. Wind turbine description}

With a hub height of $32.5 \mathrm{~m}$ and a rotor diameter of $27 \mathrm{~m}$, the three-bladed, pitch-regulated, $225 \mathrm{~kW}$ Vestas V27 turbine (pictured in Fig. 3) was chosen for the measurement campaign because its design is similar to that of larger utility-scale turbines, yet it is small enough to allow for rapid scanning of its induction zone while keeping the lidars relatively close to the turbine. A list of the parameters for the V27 is included in Table I. For wind speeds below $\sim 5.5 \mathrm{~m} / \mathrm{s}$, power is generated using a $50 \mathrm{~kW}$ generator and the rotor's rotational speed is fixed at $33 \mathrm{rpm} .{ }^{16}$ At higher wind speeds, power is generated using the $225 \mathrm{~kW}$ generator with a constant rotational speed of $43 \mathrm{rpm}$. When wind speeds exceed $14 \mathrm{~m} / \mathrm{s}$, rated wind speed for the V27, blade pitch control is used to regulate power capture.

The published power curve for the V27 turbine ${ }^{16}$ is plotted in Fig. 4(a). Using data collected during early testing of the V27 at Ris $\varnothing,{ }^{17}$ the estimated $C_{P}$ curves based on electrical power production and mechanical power production (the latter relying on modifying the electrical $C_{P}$ curve using measurements of the generator transmission efficiency) are plotted in Fig. 4(b). Figure 4(b) also includes the estimated axial induction factor curve for the V27 calculated by solving for $a$ using the mechanical $C_{P}$ curve and Eq. (2). Although two solutions for $a$ exist for a given $C_{P}$ value, it was assumed that the turbine operates at induction factors below the optimal value of $1 / 3$. Higher values of $a$ would cause unnecessarily high rotor thrust. Note that values of mechanical $C_{P}$ above the Betz limit of 16/27, and thus undefined axial induction factors, are present at low wind speeds. These unrealistic $C_{P}$ values are due to errors in the estimation of the generator efficiency for the $50 \mathrm{~kW}$ generator used when the wind speed is below $5.5 \mathrm{~m} / \mathrm{s}$, specifically errors in the measurement of the applied mechanical torque. ${ }^{17}$ At low wind speeds, the measured generator efficiency is very low (approaching 20\%-30\%) and estimates of mechanical $C_{P}$ are highly susceptible to small efficiency estimation errors. But as the wind speed increases and the $225 \mathrm{~kW}$ generator is utilized the estimated generator efficiency asymptotically approaches $\sim 95 \%$ and the mechanical $C_{P}$ estimates are expected to become more accurate. It should be noted that only lidar measurement cases with mean wind speeds above $5.5 \mathrm{~m} / \mathrm{s}$, yielding reasonable $C_{P}$ values, are analyzed in this paper. However, the induction factor curve based on the estimated mechanical $C_{P}$ shown in Fig. 4(b) is primarily meant to serve as a rough guideline during data analysis so that lidar measurement periods with mean wind speeds corresponding to a wide range of axial induction factors can be analyzed. Finally, the method of estimating the induction factor using Eq. (2) is only an approximation, relying on the simple actuator disk theory.
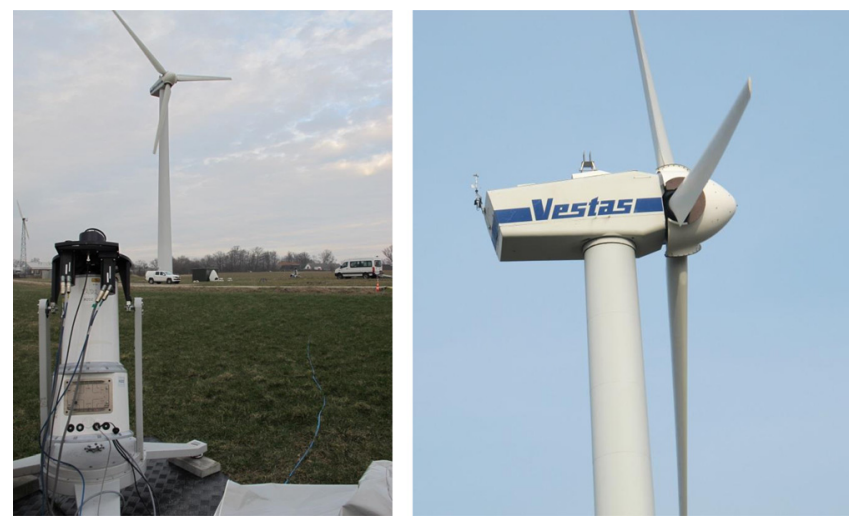

FIG. 3. Lidar 2 positioned in the field with V27 wind turbine in the background (left). Close-up of V27 nacelle and rotor (right). 
TABLE I. Summary of Vestas V27 wind turbine parameters. Values selected from Ref. 16.

\begin{tabular}{lc}
\hline \hline Parameter & Value \\
\hline Type & 3 bladed, upwind oriented \\
Rated power & $225 \mathrm{~kW}$ \\
Rotor diameter & $27 \mathrm{~m}$ \\
Hub height & $32.5 \mathrm{~m}$ \\
Power regulation & Pitch control \\
Rotor speed, $50 \mathrm{~kW}$ generator $(\mathrm{U} \leq 5.5 \mathrm{~m} / \mathrm{s})$ & $33 \mathrm{rpm}$ \\
Rotor speed, $225 \mathrm{~kW}$ generator $(\mathrm{U} \geq 5.5 \mathrm{~m} / \mathrm{s})$ & $43 \mathrm{rpm}$ \\
Cut-in wind speed & $3.5 \mathrm{~m} / \mathrm{s}$ \\
Rated wind speed & $14 \mathrm{~m} / \mathrm{s}$ \\
Cut-out wind speed & $25 \mathrm{~m} / \mathrm{s}$ \\
\hline \hline
\end{tabular}

\section{B. Lidar system}

The short-range WindScanner lidar system (see Fig. 3) developed by DTU Wind Energy is used to measure the wind inflow to the V27 turbine. The short-range WindScanner system consists of three continuous-wave (CW) lidars developed by ZephIR that have been modified with dual-prism scanning systems capable of orienting the beam in any direction within $60^{\circ}$ of the adjustable center axis. ${ }^{14,18}$ Two motors are used to orient the beam while a third motor is used to control the focus distance of the lidars between $10 \mathrm{~m}$ and $200 \mathrm{~m}$. The short-range WindScanners are capable of sampling the wind speed at $400 \mathrm{~Hz}$, but a sample rate of $100 \mathrm{~Hz}$ is used for this campaign. All three WindScanner lidars can be synchronized to scan the same pattern in space simultaneously allowing for line-of-sight measurements from three unique directions, and therefore the ability to solve for the three wind speed components.

Rather than measuring the line-of-sight velocities at precisely the point where the lidars are focused, some volume averaging, or "range weighting" occurs along the beam. A mathematical description of the approximate line-of-sight, range-weighted measurement $v_{\text {los }}$ for a focus distance $F$ and a beam direction unit vector $\vec{\ell}=\left[\ell_{x}, \ell_{y}, \ell_{z}\right]$ is provided in the following equation:

$$
v_{l o s}(F, \vec{\ell})=-\int_{0}^{\infty}\left(\ell_{x} u(R)+\ell_{y} v(R)+\ell_{z} w(R)\right) W(F, R) d R .
$$

The longitudinal $x$ axis is aligned with the mean wind direction, $y$ is defined as the horizontal transverse direction, perpendicular to the $x$ axis, and $z$ is the vertical direction. The longitudinal

(a)

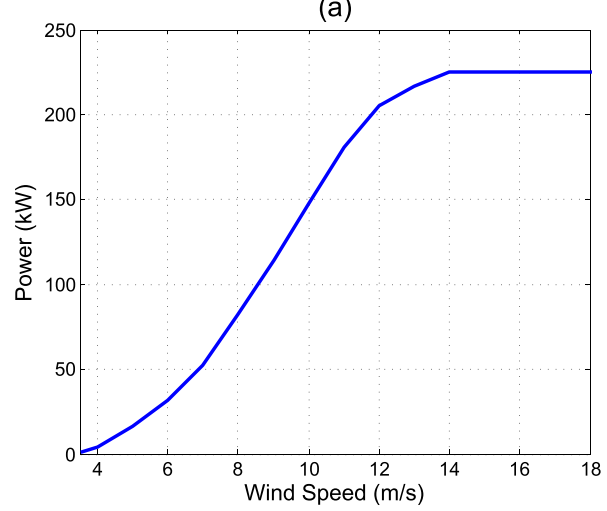

(b)

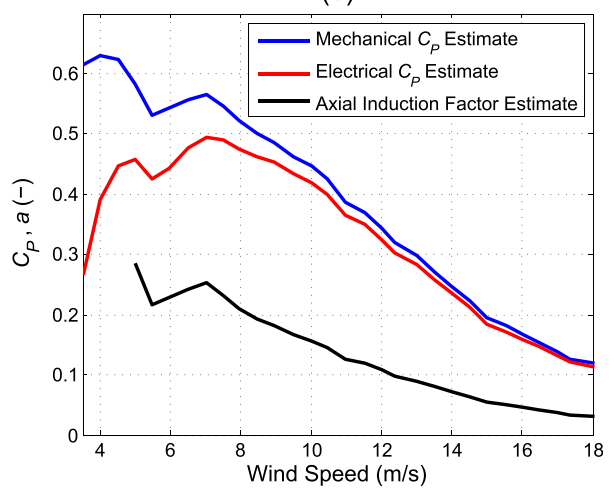

FIG. 4. (a) Vestas V27 power curve. Values obtained from Ref. 16. (b) Estimated coefficient of power curves for generated electrical power and mechanical power produced by the low-speed shaft (values obtained from Ref. 17) as well as estimated axial induction factor curve using Eq. (2). Due to uncertainty in the estimation of generator efficiency, ${ }^{17}$ the mechanical $C p$ curve is assumed to be unreliable for wind speeds below $5.5 \mathrm{~m} / \mathrm{s}$. 
$(u)$, transverse $(v)$, and vertical $(w)$ wind components correspond to the $x, y$, and $z$ directions, respectively. To follow the convention that lidar measurements, when pointing into the wind direction, are detected as positive line-of-sight velocities, the negative sign appears in Eq. (5). The lidar's range weighting function $W(F, R)$, for focus distance $F$, describes the weighting applied to the radial velocities along the beam as a function of the range $R$ along the beam. For a $\mathrm{CW}$ lidar, the range weighting function can be approximated as

$$
W(F, R)= \begin{cases}\frac{1}{\pi} \frac{Z_{R}}{Z_{R}^{2}+(R-F)^{2}}, & \text { if } \quad R \geq 0 \\ 0, & \text { otherwise }\end{cases}
$$

where $Z_{R}$ is the Rayleigh length, defined as the distance from the focus point where the laser beam's cross-sectional radius has doubled. ${ }^{19}$ The Rayleigh length can be written as

$$
Z_{R}=\frac{\lambda F^{2}}{\pi} \frac{a_{2}^{2}}{a^{2}}
$$

where $\lambda$ is the laser wavelength and $a_{2}$ is the $e^{-2}$ intensity radius of the laser beam at the lidar telescope. For the modified ZephIR lidars used in the WindScanner system, $\lambda=1.565 \mu \mathrm{m}$ and $a_{2}=28 \mathrm{~mm}$. The resulting full-width-at-half-maximum (FWHM) width of the range weighting function, centered at the focus point, can be approximated as $2 \cdot Z_{R}=0.0013 \cdot F^{2}$. For this measurement campaign, the focus distances during the lidar scans range from approximately $33 \mathrm{~m}$ to $75 \mathrm{~m}$ resulting in range weighting FWHM widths between approximately $1.5 \mathrm{~m}$ and $7 \mathrm{~m}$.

\section{Lidar data processing}

At every time sample, a Doppler velocity spectrum is provided by each lidar. Each Doppler spectrum is formed by computing the discrete Fourier transform of the backscattered light sampled at a rate of $100 \mathrm{MHz}$ and contains 256 frequency bins, where the width of each frequency bin represents a velocity change of $0.153 \mathrm{~m} / \mathrm{s}$. To allow the ability to distinguish between negative and positive velocities, the Doppler spectrum detected by a WindScanner lidar is shifted by $27 \mathrm{MHz}$ using an acousto-optic modulator (AOM) so that frequencies from $0 \mathrm{~Hz}$ to $27 \mathrm{MHz}$ represent negative velocities and frequencies from $27 \mathrm{MHz}$ to $50 \mathrm{MHz}$ represent positive velocities. ${ }^{20}$ However, low-frequency noise caused by laser light leakage in the lidar optics and mechanical vibrations in the optical fiber, which is shifted by the AOM to the frequency band near $27 \mathrm{MHz}$, makes it difficult to detect velocities between approximately $-0.5 \mathrm{~m} / \mathrm{s}$ and $0.5 \mathrm{~m} / \mathrm{s}$.

Approximately 2000 individual 256-bin Doppler spectra are averaged together during the $\sim 10 \mathrm{~ms}$ sampling period to reduce noise; for longer sampling periods, noise suppression is higher. Because of range weighting, the Doppler spectrum contains energy at frequencies corresponding to all of the velocities detected along the beam, weighted by $W(F, R)$. After subtracting the mean background noise spectrum, only frequency bins containing energy in excess of some multiple of the standard deviation of the remaining noise floor are considered in the final Doppler spectrum..$^{20}$ In this investigation, threshold values of either 3 or 5 standard deviations of the noise floor are used, depending on the lidar scan. Once the background noise is removed from the Doppler spectrum, a single value of the line-of-sight velocity detected by the lidar is estimated. For example, the velocity bin containing the maximum amount of energy or the velocity representing the centroid of the Doppler spectrum could be used to estimate the lineof-sight velocity. In the absence of Doppler spectrum noise, the centroid method yields the mean velocity detected in the lidar probe volume, which is the desired quantity to represent the line-of-sight velocity. Due to the sensitivity of this approach to spurious noise in the spectra far from the true mean line-of-sight velocity, however, the more robust approach of identifying the median velocity, i.e., the velocity that half of the energy in the Doppler spectrum lies above and half the energy lies below, is used. In other words, in the presence of spurious Doppler 
spectrum noise, the velocity estimate using the chosen median method tends to more accurately represent the desired mean line-of-sight velocity than the centroid method. More information about estimating the line-of-sight velocity from a Doppler spectrum is provided by Angelou et $a .^{20}$

Once the line-of-sight velocities for the three lidars focusing at the same point in space are known, the measurements are used to estimate the orthogonal $u, v$, and $w$ wind components of interest. This estimation is achieved by solving the inverse detection problem to find the $u, v$, and $w$ components that would have produced the three line-of-sight measurements. In reality, due to the range-weighting effect in Eq. (5), each of the lidar-attained line-of-sight measurements represents a spatial average of the instantaneous wind velocity vector projected onto the beam direction. Therefore, it is not possible to determine the exact velocity components at the focus point. However, volume-averaged estimates of the $u, v$, and $w$ components at the common focus point can be solved for using

$$
\left[\begin{array}{c}
u \\
v \\
w
\end{array}\right]=-\left[\begin{array}{lll}
\ell_{x, 1} & \ell_{y, 1} & \ell_{z, 1} \\
\ell_{x, 2} & \ell_{y, 2} & \ell_{z, 2} \\
\ell_{x, 3} & \ell_{y, 3} & \ell_{z, 3}
\end{array}\right]^{-1}\left[\begin{array}{l}
v_{l o s, 1} \\
v_{l o s, 2} \\
v_{l o s, 3}
\end{array}\right],
$$

where $\left[\ell_{x, i}, \ell_{y, i}, \ell_{z, i}\right]$ and $v_{l o s, i}$ are the beam direction unit vector and measured line-of-sight velocity, respectively, for lidar $i$. It is desirable to choose lidar positions and scan patterns that result in a well-conditioned matrix in Eq. (8) to minimize the sensitivity of the $u, v$, and $w$ component estimates to errors in the line-of-sight measurements and beam direction vectors.

\section{Lidar scan scenarios}

Three lidar scan patterns were used to measure wind velocities in the induction zone: two used to measure the time-averaged mean velocity components upstream of the rotor and one used to measure turbulence characteristics. The lidar positions were chosen to allow measurements for a $60^{\circ}$ range of wind directions, up to $30^{\circ}$ away from an ideal wind direction. A $60^{\circ}$ range of acceptable wind directions allows a trade-off between optimizing for a single wind direction and enabling enough data to be collected during the campaign given the variability in wind direction. After analyzing the distribution of wind directions encountered by the V27, it was decided to allow measurements for directions from $221^{\circ}$ to $281^{\circ}$, with $251^{\circ}$ as the ideal direction. In practice, only lidar scans intended for wind directions between $251^{\circ}$ and $280^{\circ}$ were performed during the campaign (see Fig. 2).

When choosing the lidar positions, an additional guideline was the desire to avoid measuring line-of-sight velocities close to 0 , which, as explained in Section IIC, are difficult to accurately measure. Therefore, the lidar positions and scan patterns were chosen to avoid measuring perpendicular to the intended mean wind directions. To minimize volume averaging due to range weighting, yielding measurement scans with higher spatial resolution, the lidars were placed as close to the intended scan pattern locations as possible while avoiding measurements perpendicular to the mean wind direction. To minimize the lidar focus distances, resulting in less range weighting, only locations up to roughly $1.5 \mathrm{D}$ upstream of the turbine were scanned. Furthermore, only the area upstream of the left side of the rotor (when looking upstream) was measured, under the assumption that the behavior of the induction zone is symmetric about the rotor center. The chosen lidar positions are shown in Figure 2(b). Lidar 1 is positioned upstream and to the right of the intended scan area, lidar 2 is positioned upstream and to the left, and lidar 3 is located downstream of the scan area resulting in a diversity in measurement angles throughout the scan. While the lidar positions were not chosen to produce measurement matrices (see Eq. (8)) with condition numbers below a specific threshold, the diverse measurement angles help reduce the sensitivity of the $u, v$, and $w$ component estimates to measurement and beam direction errors.

The majority of the lidar scans performed during the measurement campaign were horizontal planes at hub height extending from $0.1 \mathrm{D}(2.7 \mathrm{~m})$ downstream of the rotor to $1.6 \mathrm{D}$ 
upstream and from $1 / 8 \mathrm{D}$ beyond the left edge of the rotor, when looking upstream, to $1 / 8 \mathrm{D}$ to the right of the hub. The scan is performed by repeating a 10 -s pattern covering the area of interest, which is plotted in Fig. 5(a) for a wind direction, and therefore $x$ axis direction, of $251^{\circ}$. Note the positions of the lidars in Fig. 5, plotted as red points, which result in no measurements perpendicular to the wind direction of $251^{\circ}$. For wind directions far enough above $251^{\circ}$, however, lidar 2 measures perpendicular to the wind direction during portions of the scan. Measurement periods with such wind directions are still analyzed in this study, but the distance upstream of the rotor for which data can be analyzed is limited. In terms of rotor diameters $D$, the 10 -s scan is defined as

$$
\vec{x}(t)=\left\{\begin{array}{l}
x(t)=-0.85 D \sin (2 \pi t)-0.75 D \\
y(t)=0.375 D \sin (2 \pi t / 10)-0.25 D \\
z(t)=32.5 \mathrm{~m},
\end{array}\right.
$$

where sinusoidal scan positions are used to reduce the acceleration of the WindScanners' prism and focus motors. This scan pattern is used to form 10-min time-averaged mean values of the wind velocities to reveal the behavior of the longitudinal $(u)$, radial, and tangential wind speeds at different points in the induction zone. The radial wind speed is defined as the wind speed component oriented away from the rotor center, perpendicular to the longitudinal direction, and the tangential wind speed is defined as the component of the wind aligned with the rotational direction of the rotor, i.e., perpendicular to the radial component in the $y z$ plane. Thus, for the horizontal plane scan, the radial wind speed is equivalent to the $v$ component and the tangential

(a)

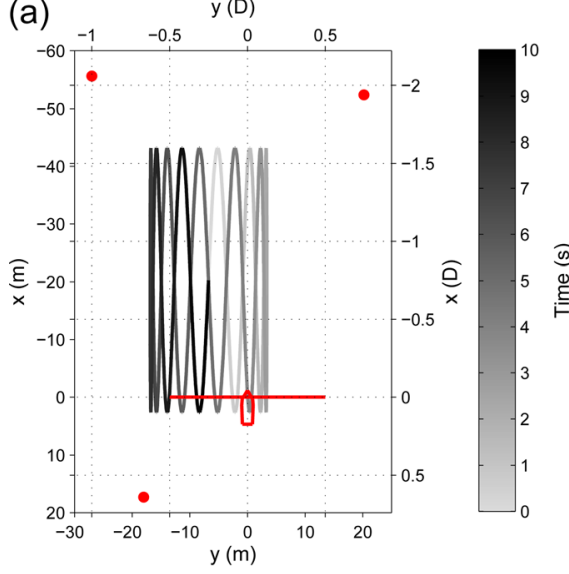

(b)

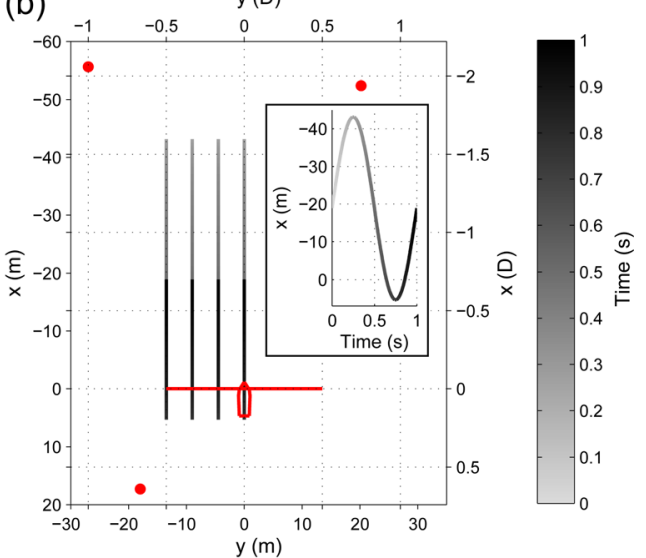

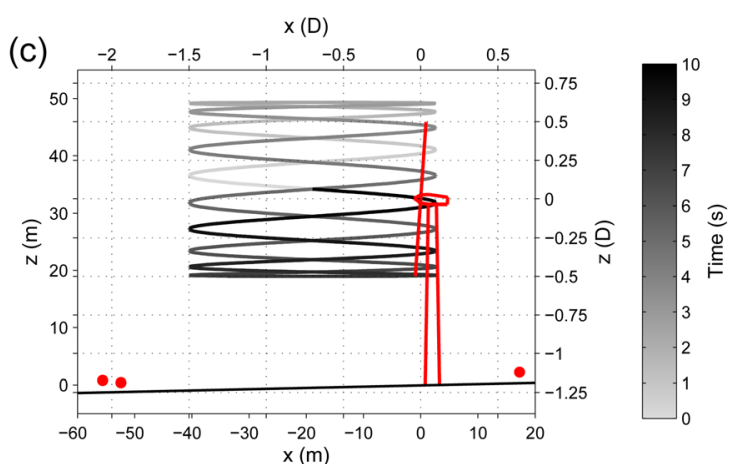

FIG. 5. Lidar scan patterns used to measure the induction zone. (a) Top view of horizontal scan plane at hub height. (b) Top view of longitudinal line scans at hub height for radial positions at $0,1 / 6,1 / 3$, and 1/2 $D$. An inset plot shows the longitudinal scan position as a function of time. (c) Side view of vertical scan plane located at the center of the rotor in the transverse direction $(y=0)$. The lidar positions are indicated by the red points. 
wind speed is equivalent to the $w$ component. All scan patterns described in this section can be adjusted so that the $x$ axis is aligned with any wind direction between $221^{\circ}$ and $281^{\circ}$.

A second scan pattern, consisting of a vertical scan plane, was used to measure timeaveraged mean velocities as a function of longitudinal and radial position in the induction zone as well. The scan plane extends from $0.1 \mathrm{D}$ downstream of the rotor to $1.5 \mathrm{D}$ upstream of the rotor and from the bottom of the rotor to $1 / 8 \mathrm{D}$ above the top of the rotor, located at the center of the rotor in the transverse direction, i.e., intersecting the hub position. This 10-s vertical scan pattern, which allows measurements upstream of the entire rotor diameter, is shown in Fig. 5(c) and is defined as

$$
\vec{x}(t)=\left\{\begin{array}{l}
x(t)=-0.85 D \sin (2 \pi t)-0.75 D \\
y(t)=0 \mathrm{~m} \\
z(t)=0.5625 D \sin (2 \pi t / 10)+0.0625 D+32.5 \mathrm{~m} .
\end{array}\right.
$$

As with the horizontal scan plane, this 10-s pattern is repeated to form 10-min time averages of the measured inflow.

The third scan pattern used during the measurement campaign is designed to measure the turbulence characteristics in the induction zone. Similar to the horizontal scan plane, this scan pattern measures the wind upstream of the left side of the rotor at hub height, but along lines perpendicular to the rotor plane extending from $0.2 \mathrm{D}$ downstream of the rotor to $1.6 \mathrm{D}$ upstream at different radial positions. The line scans are performed at radial distances of $0 \mathrm{D}$ (the hub location), $1 / 6 D, 1 / 3 D$, and 1/2 D. Each complete line scan, pictured in Fig. 5(b), is a 1 -s sinusoidal pattern defined for radial positions $i \in\{1,2,3,4\}$ as

$$
\vec{x}(i, t)=\left\{\begin{array}{l}
x(t)=-0.9 D \sin (2 \pi t)-0.7 D \\
y(i, t)=-(i-1) D / 6 \\
z(t)=32.5 \mathrm{~m}
\end{array}\right.
$$

During the field measurements, the 1-s line scans were repeated for $2.5 \mathrm{~min}$ at each radial position before advancing to the next position, forming a sequence which was repeated every $10 \mathrm{~min}$. In contrast to the horizontal and vertical plane scans, these line scans were used to determine wind velocity time series, instead of mean values. Time series at various longitudinal distances from the rotor plane are formed by grouping the measurements by their $x$ positions using a bin size of $1 \mathrm{~m}$ and interpolating the resulting data for each bin to form $1 \mathrm{~Hz}$ time series.

\section{E. Lidar measurement error sources}

Several sources of potential measurement error exist in the lidar scenarios employed in this research, which are discussed in this section along with their implications for measurement accuracy. However, the underlying accuracy of the lidar scan directions and focus distances is very high. The pointing uncertainty of the WindScanners is approximately $0.2^{\circ}$, while the focus distance uncertainty is between $0.15 \mathrm{~m}$ and $0.7 \mathrm{~m}$ for the range of focus distances used in the chosen scan patterns. Furthermore, the three lidars are synchronized to within $1.7 \mathrm{~ms}$ of uncertainty. In practice, the Doppler spectra and the corresponding scan locations reported by the lidars are synchronized with an uncertainty of less than half of a sampling period, or $\sim 5 \mathrm{~ms}$, yielding a maximum measurement location uncertainty of $\sim 1.5 \mathrm{~m}$ (or a maximum effective pointing uncertainty of less than $1^{\circ}$ ) during the fastest part of the scan.

One source of potential measurement error is the process through which the $u, v$, and $w$ wind speed components are derived from the three line-of-sight velocities using Eq. (8). In order to reduce the impact that errors in the line-of-sight measurements have on the estimated $u, v$, and $w$ components, the individual elements of the inverse detection matrix that the line-ofsight velocity vector is multiplied by in Eq. (8) should be kept small. Specifically, the 
sensitivity to line-of-sight velocity errors can be quantified using the norm of each row of the matrix. The norm of a particular row represents the maximum error that a unity-magnitude vector of line-of-sight measurement errors can cause when estimating the corresponding $u, v$, or $w$ component. To minimize the sensitivity of the inverse detection process, the three lidar beams should intersect at $90^{\circ}$ angles at the focus point, yielding norms of 1 for each of the three inverse detection matrix rows. But since the lidars are used to scan across a large area in this study, the intersection angles cannot always be maintained close to $90^{\circ}$. In practice, the intersection angles range between $39^{\circ}$ and $104^{\circ}$ during the horizontal plane scans and line scans, with a mean intersection angle of $78^{\circ}$, while for the vertical plane scan the intersection angles vary between $35^{\circ}$ and $130^{\circ}$ with a mean value of $76^{\circ}$. These intersection angles produce maximum norms of $1.09,2.07$, and 1.6 for the matrix rows used to estimate the $u, v$, and $w$ components, respectively, for the horizontal plane and line scans, with maximum norms of 1.22, 2.29, and 2.12 for the vertical scan plane. Thus, the sensitivity of the inverse detection process to line-of-sight measurement errors is close to the optimal value for the $u$ component, but roughly twice as high for the $v$ component and 1.6-2.1 times as high for the $w$ component. Therefore, it is expected that the $v$ components will contain the most estimation error followed by the $w$ components, with the $u$ component estimates suffering the least.

As mentioned earlier, the FWHM of the lidar range weighting function varies between roughly $1.5 \mathrm{~m}$ and $7 \mathrm{~m}$ during the scan patterns that are employed in this research. This range weighting, or volume averaging, inherent in the lidar measurements acts as a significant source of potential measurement error. For estimates of the time-averaged mean velocities at different points in the induction zone using the horizontal and vertical scan planes, lidar range weighting can cause a bias in the mean velocity estimates when the true mean wind speeds are a nonlinear function of the location in space. If the mean wind speeds vary linearly in space, on the other hand, such as for the case of linear vertical shear, then the higher (or lower) wind speeds detected along the beam on one side of the focus point will cancel out the lower (or higher) wind speeds detected on the other side, creating zero bias. But as explained by Clive, ${ }^{21}$ range weighting can produce a bias in the mean wind speed estimates for a nonlinear logarithmic wind profile, although the bias is estimated to be less than $0.5 \%$ of the true wind speed. The induction zone produces a nonlinear spatial distribution of wind speeds as well, which can produce a mean velocity measurement bias. Range weighting along the longitudinal direction is expected to cause only a very small bias, similar to the bias created by nonlinear shear, but the spatial averaging in the wind turbine's radial direction is expected to be more significant. For example, since the velocity reductions in the induction zone are greater near the center of the rotor than near the rotor's edge ${ }^{4}$ range-weighted measurements from a lidar focused at a point along the turbine's symmetry axis will be positively biased by the higher mean wind speeds detected along the beam away from the focus point.

During the line scans used to create time series for determining turbulence statistics in the induction zone, range weighting introduces a spatial filtering effect that reduces the high frequency content of the velocity time series. As discussed by Simley et al., ${ }^{22}$ when the lidar beam direction is aligned with the longitudinal wind direction, the range weighting function can be interpreted as a filter that is convolved with the wind speed time series producing a low-pass filtered velocity measurement. For the focus distances required for the line scans, which range between $36 \mathrm{~m}$ and $70 \mathrm{~m}$, the FWHM values of the range weighting functions lie between $1.7 \mathrm{~m}$ and $6.2 \mathrm{~m}$ and the $-3 \mathrm{~dB}$ corner frequency of the equivalent spatial low-pass filters varies between $\sim 0.11 \mathrm{rad} / \mathrm{m}$ and $\sim 0.43 \mathrm{rad} / \mathrm{m}$. However, for the scan patterns used in this research the lidar beam directions are never aligned with the longitudinal wind direction and therefore range weighting cannot be analyzed as a simple one-dimensional filtering operation. Furthermore, range weighting along the three lidar beams produces an effective 3D spatial filtering operation, which is discussed by Carbajo Fuertes et al. ${ }^{12}$ for the case of pulsed lidar measurements where the range weighting functions are equivalent irrespective of the measurement range. Mann et al. ${ }^{11}$ presented a method for calculating the transfer function of the effective 3D spatial filter using numerical integration, which requires knowledge of the power spectra and spatial coherence of the turbulence. Further complicating the analysis of the effect of range weighting on 
the turbulence measurements performed in this research is the fact that the beam intersection angles as well as the lidar focus distances, and thus the range weighting functions, are constantly changing throughout the scan, causing varying filtering effects at different locations in the induction zone.

The amount of volume averaging that accompanies the measurements presented in this paper is summarized by projecting the three lidars' range weighting FWHM distances onto their respective lidar direction vectors. From the three FWHM projections, the maximum distance spanned in the $x$ direction is denoted $\delta_{x}$ and approximates the amount of spatial averaging along the $x$ direction. Similarly, $\delta_{y}$ and $\delta_{z}$ are defined as the maximum distances in the $y$ and $z$ directions spanned by the three lidars' FWHM projections, and represent the amount of spatial averaging in the transverse and vertical directions, respectively. A simple definition of the amount of volume averaging for a measurement is given by the product $\delta_{x} \delta_{y} \delta_{z}$. For the results presented in Figs. 6-15, the amount of volume averaging is indicated by the minimum and maximum probe volumes $\delta_{x} \delta_{y} \delta_{z}$ that occur across the measurement domains as well as the minimum and maximum $\delta_{x}, \delta_{y}$, and $\delta_{z}$ values individually.

\section{SUMMARY OF LIDAR MEASUREMENTS}

Data were collected using the three different scan patterns for measurement periods ranging from 40 min to $20 \mathrm{~h}$ during 12 days from mid March to early May 2014 at the Risø campus. Measurements using the horizontal scan at hub height, which constitute the majority of the acquired data, were performed on 9 separate days. The vertical scan plane was employed on three separate days and the line scan pattern was used during a single day. In order to identify acceptable data for analysis from the roughly $100 \mathrm{~h}$ of acquired measurement data, all 10-min periods when the intended wind direction of the lidar scan differs by the mean wind direction reported by the met mast by more than $15^{\circ}$ were discarded, leaving roughly $35 \mathrm{~h}$ of acceptable measurements. For the horizontal scan at hub height, six example 10-min periods were chosen for analysis in order to reveal how the behavior of the induction zone varies over a wide range of mean wind speeds, and thus axial induction factors. The six measurement periods consist of wind speeds representing axial induction factors between $\sim 0.25(U=6.89 \mathrm{~m} / \mathrm{s})$ and the minimum induction factor that occurred during all acceptable scan periods $(\mathrm{a}=\sim 0.041$, $U=16.29 \mathrm{~m} / \mathrm{s}$ ) in wind speed intervals of approximately $1-3 \mathrm{~m} / \mathrm{s}$. By visually inspecting the met mast and lidar data, a 10-min period for each desired wind speed interval was selected by identifying the period with the lowest error between mean wind direction and the chosen lidar scan direction, while maintaining low wind speed and direction variability. Because very few lidar scans were acquired using the vertical plane scan and line scan patterns, due to the schedule constraints of the campaign, only a small number of scan periods for these categories are analyzed and results for a wide range of mean wind speeds are not included.

A summary of all the lidar scans that were chosen for analysis in this study is provided in Table II. Measurement cases 1-7 are 10-min horizontal plane scans chosen from the collected data to provide a range of mean wind speeds and thus axial induction factors. Case 7 consists of measurements performed when the V27 was stopped, providing a reference scan. Case 8 contains one of the few satisfactory 10-min measurement periods using the vertical scan plane. Most of the remaining vertical scan plane measurement data occurred when the wind direction and scan direction differed by $\sim 10^{\circ}$ or more. Cases $9-16$ consist of 2.5 min line scan periods. Cases 9-12 represent successive line scans at radial positions of $1 / 6 D(y=-4.5 \mathrm{~m}), 1 / 3 D$ $(y=-9 \mathrm{~m}), 1 / 2 D(y=-13.5 \mathrm{~m})$, and at the hub location $(y=0)$ as do cases $13-16$, but for higher wind speeds. The similar sequences of scan cases 9-12 and 13-16 are both analyzed to help confirm the identified trends, since each case contains only a short measurement period.

The Scan Direction column in Table II indicates the wind direction that the $x$ axes of the scan patterns were aligned with in anticipation of the true wind direction. The Wind Direction column contains the actual mean wind directions present during the scan periods, calculated from the lidar measurements. It is difficult to determine the true freestream mean wind speeds from the lidar measurements, given that the farthest measurement locations from the turbine are 
TABLE II. Summary of lidar measurement cases. Cases 1-8 consist of 10 min measurement periods while cases 9-16 are 2.5 min scans. Turbulence intensity and wind direction standard deviation are calculated using met tower measurements at a height of $32 \mathrm{~m}$. Case 7 was performed with the turbine stopped.

\begin{tabular}{|c|c|c|c|c|c|c|c|c|c|c|}
\hline Case & $\begin{array}{l}\text { Date and time } \\
\text { (all 2014) }\end{array}$ & Scan type & $\begin{array}{c}\text { Scan } \\
\text { direction } \\
(\mathrm{deg})\end{array}$ & $\begin{array}{c}\hat{U}_{\infty} \\
(\mathrm{m} / \mathrm{s})\end{array}$ & $\hat{a}$ & $\begin{array}{c}\text { Wind } \\
\text { direction } \\
(\mathrm{deg})\end{array}$ & $\begin{array}{l}\text { Wind Dir. } \\
\text { Std. Dev. } \\
(\text { deg })\end{array}$ & $\begin{array}{l}\text { Turbulence } \\
\text { intensity } \\
(\%)\end{array}$ & $z / L$ & $\frac{U_{45}-U_{18}}{U_{32}}$ \\
\hline 1 & March 17, 10:57 & $x y$ plane & 275 & 16.29 & 0.041 & 289.5 & 4.8 & 8.9 & -0.31 & 0.079 \\
\hline 2 & March 18, 12:00 & $x y$ plane & 251 & 8.93 & 0.18 & 254.8 & 5.1 & 9.0 & -4.39 & 0.12 \\
\hline 3 & March 18, 14:09 & $x y$ plane & 251 & 10.01 & 0.16 & 260.2 & 5.0 & 11.0 & -0.54 & 0.12 \\
\hline 4 & March 19, 13:32 & $x y$ plane & 260 & 11.49 & 0.12 & 259.1 & 4.9 & 9.4 & -0.49 & 0.099 \\
\hline 5 & March 19, 16:18 & $x y$ plane & 260 & 13.26 & 0.085 & 263.4 & 6.1 & 12 & 0.047 & 0.088 \\
\hline 6 & March 21, 10:44 & $x y$ plane & 251 & 6.89 & 0.25 & 250.8 & 5.0 & 10.6 & -1.55 & 0.087 \\
\hline 7 & March 21, 12:09 & $x y$ plane & 251 & 5.67 & 0 & 249.8 & 7.0 & 15.2 & -3.41 & 0.056 \\
\hline 8 & April 30, 22:29 & $x z$ plane & 270 & 6.98 & 0.25 & 272.2 & 6.8 & 11.8 & 0.24 & 0.11 \\
\hline 9 & May $07,14: 05: 30$ & Line, $y=-4.5 \mathrm{~m}$ & 260 & 6.23 & 0.24 & 262.5 & 7.2 & 12.8 & -4.38 & 0.095 \\
\hline 10 & May $07,14: 08: 00$ & line, $y=-9 \mathrm{~m}$ & 260 & 6.25 & 0.24 & 260.1 & 7.0 & 11.8 & -4.38 & 0.095 \\
\hline 11 & May $07,14: 10: 30$ & Line, $y=-13.5 \mathrm{~m}$ & 260 & 5.95 & 0.23 & 264.1 & 5.5 & 10.4 & -4.38 & 0.095 \\
\hline 12 & May $07,14: 13: 00$ & Line, $y=0 \mathrm{~m}$ & 260 & 6.15 & 0.23 & 259.3 & 4.4 & 7.8 & -4.38 & 0.095 \\
\hline 13 & May $07,16: 03: 00$ & Line, $y=-4.5 \mathrm{~m}$ & 275 & 7.60 & 0.22 & 281.2 & 5.0 & 10.5 & -1.54 & 0.061 \\
\hline 14 & May $07,16: 05: 30$ & Line, $y=-9 \mathrm{~m}$ & 275 & 7.49 & 0.22 & 277.8 & 5.8 & 7.0 & -1.54 & 0.061 \\
\hline 15 & May $07,16: 08: 00$ & Line, $y=-13.5 \mathrm{~m}$ & 275 & 8.09 & 0.20 & 275.9 & 6.0 & 10.9 & -1.54 & 0.061 \\
\hline 16 & May $07,16: 10: 30$ & Line, $y=0 \mathrm{~m}$ & 275 & 7.40 & 0.23 & 278.6 & 6.5 & 9.4 & -1.54 & 0.061 \\
\hline
\end{tabular}

roughly $1.5 \mathrm{D}$ upstream of the rotor, where velocity reductions of a few percentage points of the freestream velocity are expected. Furthermore, there are occasionally significant discrepancies between the mean wind speeds measured by the nearby met mast and those detected by the lidars far upstream of the turbine. Therefore, the mean wind speeds (under the $\hat{U}_{\infty}$ column) are estimated by solving the vortex sheet theory formula (Eq. (4)) presented by Medici et al. ${ }^{4}$ and plotted in Fig. 1(a), giving the velocities upstream of the rotor center, for the freestream velocities using the measured wind speeds at either $x=-1 D$ or $x=-1.5 D$ and the estimated axial induction factors. The estimated axial induction factors (a) are based on the induction factor curve plotted in Fig. 4 and the mean velocities measured at $x=-1 D$ or $x=-1.5 D$. After applying the vortex sheet theory formula, the estimated freestream velocities only differ from the values at $x=-1 D$ or $x=-1.5 D$ by up to $3 \%$ or $1 \%$, respectively, however. Next, the $u$ component "Wind Direction Standard Deviation" and "Turbulence Intensity" columns contain values measured at a height of $32 \mathrm{~m}$ on the met tower $2.7 \mathrm{D}$ to the west of the turbine. Measurement periods with low wind direction standard deviations were selected for analysis to keep the instantaneous wind directions close to the lidar scan direction.

Finally, parameters characterizing the atmospheric boundary layer and the vertical wind shear are provided. Boundary layer stability is indicated by the Monin-Obukhov stability parameter $z / L$, calculated using met tower measurements at a height of $z=18 \mathrm{~m}$, where negative values represent unstable conditions, positive values correspond to stable conditions, and values close to zero indicate near neutral conditions. ${ }^{23}$ The Monin-Obukhov length is calculated here as

$$
L=\frac{-u_{*}^{3} \bar{\theta}}{\kappa g\left(\overline{w^{\prime} \theta^{\prime}}\right)},
$$

where $\kappa=0.4$ is the von Karman constant, $g$ represents gravitational acceleration, $\theta$ is the potential temperature, \{\}$^{\prime}$ indicates the zero-mean fluctuating component of a variable, and the friction velocity $u_{*}$ is estimated as

$$
u_{*}=\sqrt{-\left(\overline{u^{\prime} w^{\prime}}\right)}
$$


All values of $z / L$ reported in Table II were calculated using 10 -min averaging periods. Note that most of the lidar measurements were obtained during the daytime with unstable conditions (aside from case 5), while the vertical scan plane measurement period, obtained during the nighttime, contains stable conditions. Mean wind speed measurements at heights of $18 \mathrm{~m}, 32 \mathrm{~m}$, and $45 \mathrm{~m}$ (roughly corresponding to the bottom of the rotor disk, hub height, and the top of the rotor disk) on the met tower are used to calculate a vertical wind shear parameter defined as $\left(U_{45}-U_{18}\right) / U_{32}$ indicating the normalized difference in mean wind speed between the top and bottom of the rotor disk. Note that the cases investigated do not contain a wide variety of shear parameters. Therefore, the dependence of the induction zone behavior on the amount of wind shear is difficult to assess in this study.

\section{RESULTS: RADIAL AND LONGITUDINAL DEPENDENCE OF MEAN WIND SPEEDS IN THE INDUCTION ZONE}

The results in this section illustrate the measured time-varying mean longitudinal, radial, and tangential wind speeds as a function of longitudinal and radial distance in the induction zone using the horizontal and vertical plane scans discussed in Section IID.
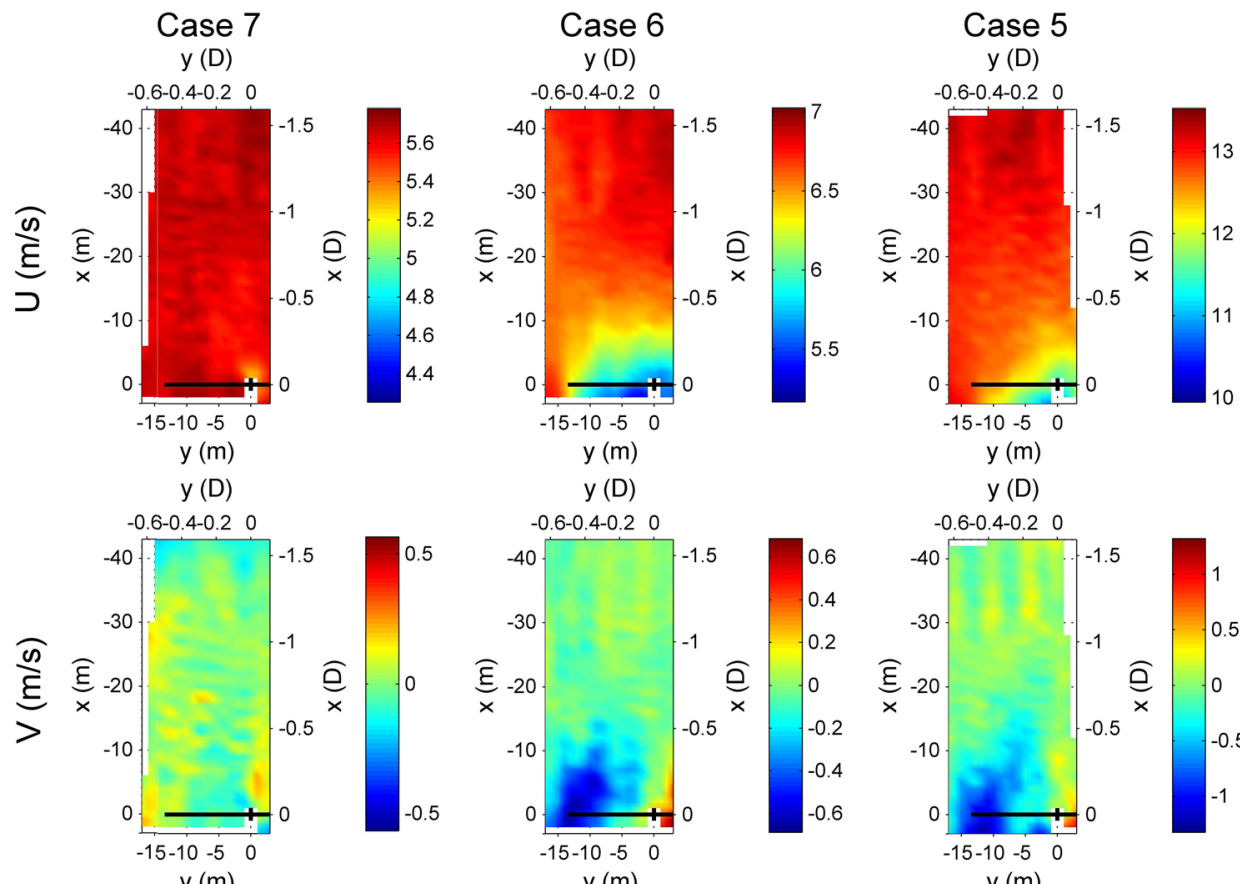

$y(D)$
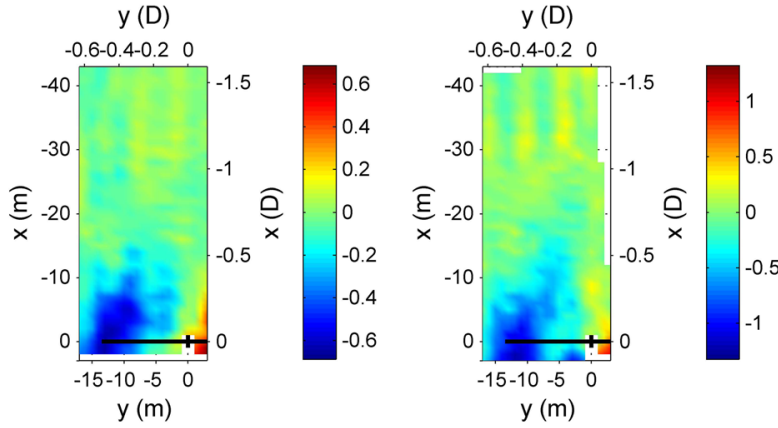

y (D)
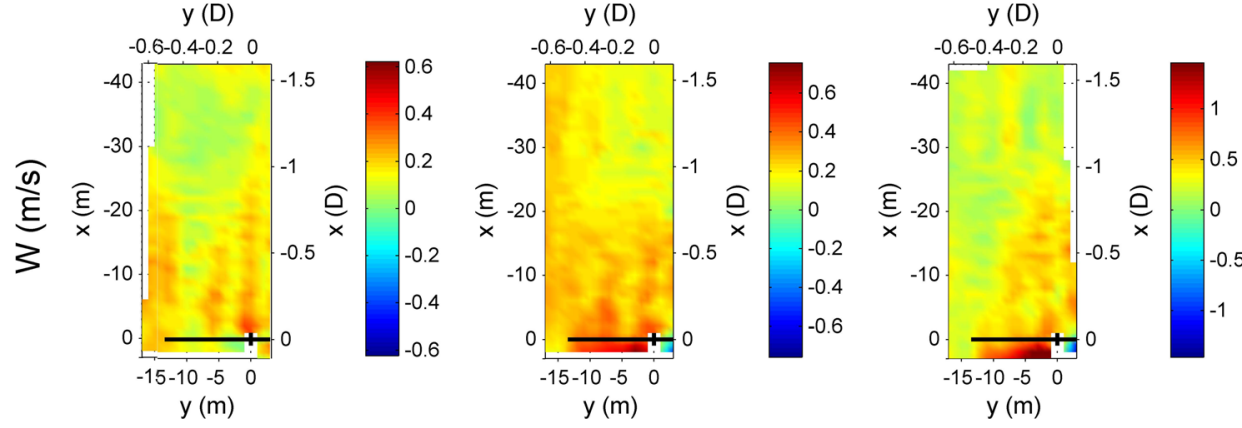

FIG. 6. Measured $U, V$, and $W$ wind components (top, middle, and bottom rows, respectively) for a horizontal scan plane at hub height for measurement cases $7\left(\hat{U}_{\infty}=5.67 \mathrm{~m} / \mathrm{s}\right.$, turbine stopped $), 6\left(\hat{U}_{\infty}=6.89 \mathrm{~m} / \mathrm{s}\right)$, and $5\left(\hat{U}_{\infty}=13.26 \mathrm{~m} / \mathrm{s}\right)$. The amount of lidar spatial averaging in the $x, y$, and $z$ directions ranges from $\delta_{x}=2.3 \mathrm{~m}$ to $\delta_{x}=5.8 \mathrm{~m}, \delta_{y}=1.3 \mathrm{~m}$ to $\delta_{y}=3.8 \mathrm{~m}$, and $\delta_{z}=2 \mathrm{~m}$ to $\delta_{z}=3 \mathrm{~m}$, respectively. The approximate lidar probe volumes $\delta_{x} \delta_{y} \delta_{z}$ range from $8.0 \mathrm{~m}^{3}$ to $54.6 \mathrm{~m}^{3}$. 


\section{A. Horizontal scan plane at hub height}

Figure 6 shows the 10-min time-averaged mean longitudinal $(U)$, radial $(V)$, and tangential $(W)$ velocities measured in the horizontal scan plane at hub height for cases 5,6 , and 7. Unless otherwise stated, the discussion of mean wind velocities in the induction zone refers to the time-averaged mean wind speeds as a function of space rather than spatially averaged wind speeds. In order of increasing mean freestream wind speed $U_{\infty}$, case 7 has an estimated wind speed of $5.67 \mathrm{~m} / \mathrm{s}$, with the wind turbine stopped to provide a reference measurement case free of rotor induction effects, case 6 has a value of $\hat{U}_{\infty}=6.89 \mathrm{~m} / \mathrm{s}$ with a relatively high estimated induction factor of $\hat{a}=0.25$, and case 5 contains an estimated freestream wind speed of $\hat{U}_{\infty}=13.26 \mathrm{~m} / \mathrm{s}$ with a relatively low $\hat{a}$ value of 0.085 . The velocity plots are formed by grouping the measurements by their $(\mathrm{x}, y)$ positions using $1 \mathrm{~m}$-by-1 $\mathrm{m}$ bins and averaging all Doppler spectra belonging to each bin. Mean line-of-sight velocities are determined from the averaged Doppler spectra at each of the bin locations and bilinear interpolation in the $x y$ plane is used to find values at bins in the scan plane for which no measurements were acquired during the scan period. The mean line-of-sight velocities at each bin location in the $x y$ plane are then transformed to the $U, V$, and $W$ components using Eq. (8) by applying the average of the slightly different individual inverse detection matrices. While this method produces slightly different results than averaging the individual $u, v$, and $w$ components formed by applying Eq. (8) to the detected line-of-sight velocities at each relevant sample period, averaging the Doppler spectra prior to determining the velocities allows the background spectral noise to be significantly reduced. Although the ability to determine velocity time series is lost, this method is well suited for estimating the mean velocities. Measurements corresponding to lidar beam directions pointing at the turbine, including the blades, are detected and removed before calculation of the velocities. Note that the scan patterns plotted in Fig. 6 are rotated so that the $x$ axis is aligned with the true wind direction rather than the original scan direction.

For measurement case 7, with the turbine stopped, there are no drastic variations in the $U$, $V$, and $W$ components within the scan plane, aside from a drop in the longitudinal wind speed near the nacelle. To allow easier visual comparison between the relative velocity variations for the three cases shown in Fig. 6, the limits of the color axes are identical across each row after normalization by the estimated mean freestream wind speed $\hat{U}_{\infty}$. For case 6 , significant longitudinal velocity deficits occur in the induction zone, especially within $0.5 \mathrm{D}$ of the rotor. The $U$ component velocity reductions are present in case 5 as well, although, as expected given the lower induction factor, they are less drastic and are also more concentrated near the center of the rotor, possibly due to lower induction at the outboard region of the blades. Consistent with the expected expansion of the wind inflow around the rotor disk, illustrated in Fig. 1, the radial $V$ components are negative (directed away from the rotor center) near the edge of the rotor for cases 5 and 6 . Thus the mean wind direction changes in that region, deflecting away from the rotor center. Significant positive vertical $W$ components can be seen behind the rotor when the turbine is operating. This behavior is expected because the blades are traveling downward on the left half of the rotor and the induced tangential velocities should be opposite the direction of rotor rotation. ${ }^{1}$ For all three cases in Fig. 6, the mean $W$ component upstream of the rotor is slightly positive $(0.1 \mathrm{~m} / \mathrm{s}$ for case $7,0.18 \mathrm{~m} / \mathrm{s}$ for case 6 , and $0.2 \mathrm{~m} / \mathrm{s}$ for case 5$)$, likely due to the gently sloping nature of the terrain between the fjord and the V27. The vertical wind speeds do not vary significantly upstream of the rotor, however, and are therefore not analyzed in depth in this study.

The velocity plots in Fig. 6 (as well as those in Fig. 12 in Section IV B) reveal longitudinal stripe patterns as well as alternating high and low velocities in the longitudinal direction which are artifacts of the lidar scan process. There are two reasons why these patterns appear. First of all, there are very minor timing errors between the lidar motor position values, used to determine the scan positions, and the corresponding Doppler spectra. This results in small offsets between the detected velocities and their reported locations in the scan plane, and has the effect of causing the detected velocities to either lead or lag the actual measurement positions. As can be seen in Figs. 5(a) and 5(c), there are alternating regions of the scan plane where the lidar 
focus positions are moving in either the positive $x$ direction or in the negative $x$ direction. The line-of-sight velocities detected by the lidars tend to either increase or decrease along the $x$ direction due to the changing lidar beam angles. As a result, depending on the $x$ direction the lidar scan is moving in, the lead or lag in the velocity measurements will cause the detected line-of-sight velocities to be either slightly higher or slightly lower than their true values, on average. These errors are then transferred to the calculated $u, v$, and $w$ components and appear as longitudinal stripes in the velocity plots. The motor position timing errors are minimized as much as possible, however, to within the sampling period of $10 \mathrm{~ms}$, and therefore cause only minor artifacts in the measurements. A second, unavoidable reason for the artifacts in the velocity plots is due to the lidars measuring different areas of the scan plane at different times. Although all regions in the scan pattern are sampled every $10 \mathrm{~s}$, the regions are measured at different times. Due to the turbulent nature of the inflow, the 10-min averages of the velocities are slightly different depending on exactly when the velocities are sampled during the $10 \mathrm{~min}$ period. Note that the alternating high and low velocities along the longitudinal direction visible in many of the plots are caused by this latter effect. The artifacts caused by different sampling times for different regions cannot be avoided, and are also relatively small when using a 10 min measurement period.

Although a period of $10 \mathrm{~min}$ was selected as a tradeoff between allowing enough time for the mean velocity measurements to converge, due to the turbulent nature of the wind inflow, and maintaining similar wind conditions throughout the duration of the scan, Fig. 7 reveals the impact of averaging time $\left(t_{\text {avg }}\right)$ on the obtained mean longitudinal wind speeds in the induction zone for measurement case 6 . Because of the spatial variability of the turbulence, in addition to the temporal variability expressed as turbulence intensity values in Table II, the salient features of the mean velocities in the induction zone are not revealed until a measurement period of at least $3 \mathrm{~min}$ is used. Note that the stripe-like velocity artifacts explained above are very prominent when shorter averaging times are used. Fig. 7 suggests that, if necessary, an averaging period of 5 min could be used with only a slight increase in mean wind speed estimation uncertainty compared to the results with 10-min periods used in this research.

Measurement cases 1-7 are used to determine how the behavior of the induction zone changes as the turbine's induction factor decreases. $U$ velocities normalized by $U_{\infty}$, determined from the horizontal plane scans, are plotted as a function of longitudinal distance from the rotor in Fig. 8 for six different radial positions from the hub location to $1 / 8 \mathrm{D}$ beyond the edge of the rotor. Cases $6,2,3,4,5$, and 1 represent wind speeds from $6.89 \mathrm{~m} / \mathrm{s}$ to $16.29 \mathrm{~m} / \mathrm{s}$ with estimated axial induction factors of $0.25,0.18,0.16,0.12,0.085$, and 0.041 , respectively, while case 7 , with the turbine stopped, acts a reference case. Note that velocities could only be calculated for case 1 up to $19 \mathrm{~m}$ upstream of the rotor because the actual wind direction of nearly $290^{\circ}$ causes lidar 2 to measure close to perpendicular to the wind direction for greater longitudinal distances, thus making it difficult to estimate the low line-of-sight velocities. In general, the velocity reductions are largest at the rotor center as well as the radial position of $1 / 8 D$ and become smaller towards the edge of the rotor until they are no longer noticeable beyond the
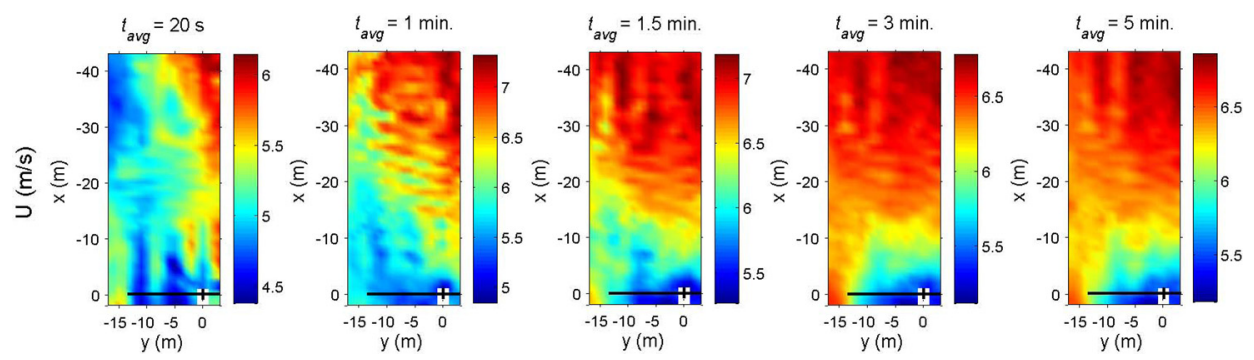

FIG. 7. Measured $U$ wind component for a horizontal scan plane at hub height for measurement case $6\left(\hat{U}_{\infty}=6.89 \mathrm{~m} / \mathrm{s}\right)$ using different scan averaging times $t_{\text {avg }}$. The amount of lidar spatial averaging in the $x, y$, and $z$ directions ranges from $\delta_{x}=2.3 \mathrm{~m}$ to $\delta_{x}=5.4 \mathrm{~m}, \delta_{y}=1.3 \mathrm{~m}$ to $\delta_{y}=3.5 \mathrm{~m}$, and $\delta_{z}=2 \mathrm{~m}$ to $\delta_{z}=3 \mathrm{~m}$, respectively. The approximate lidar probe volumes $\delta_{x} \delta_{y} \delta_{z}$ range from $8.0 \mathrm{~m}^{3}$ to $52.1 \mathrm{~m}^{3}$. 

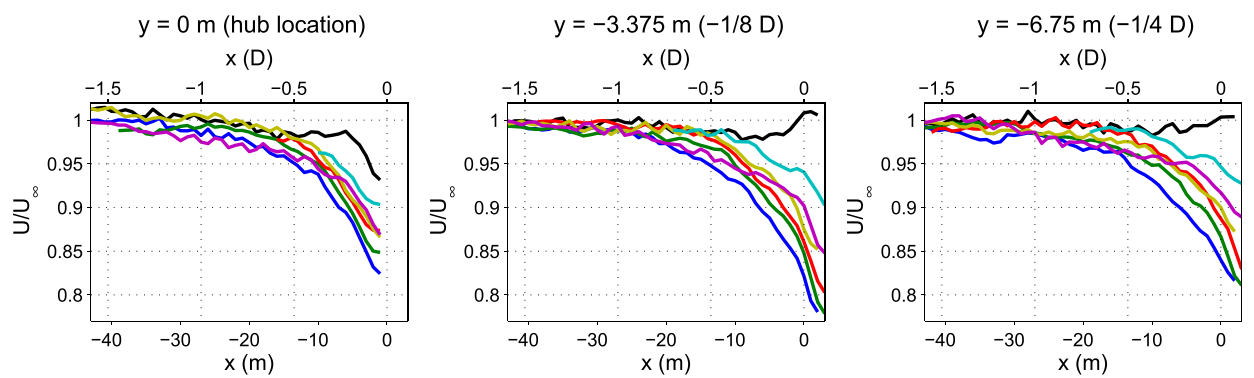

$y=-10.125 m(-3 / 8 D)$ $x(D)$
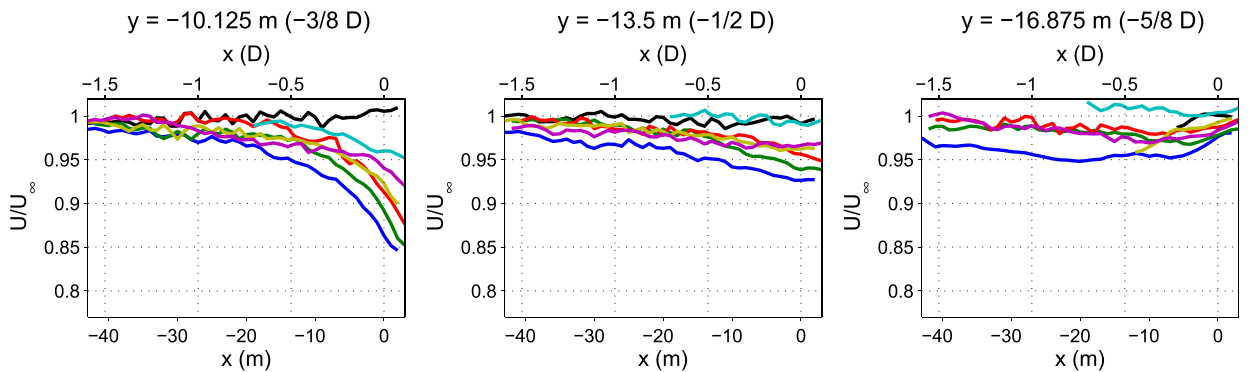

- Case 7 Case 6

Case 2

Case 3

Case 4

Case $5=$ Case 1

FIG. 8. Measured $U$ wind components as a function of radial and longitudinal position for measurement cases 7 $\left(\hat{U}_{\infty}=5.67 \mathrm{~m} / \mathrm{s}\right.$, turbine stopped $), 6\left(\hat{U}_{\infty}=6.89 \mathrm{~m} / \mathrm{s}\right), 2\left(\hat{U}_{\infty}=8.93 \mathrm{~m} / \mathrm{s}\right), 3\left(\hat{U}_{\infty}=10.01 \mathrm{~m} / \mathrm{s}\right), 4\left(\hat{U}_{\infty}=11.49 \mathrm{~m} / \mathrm{s}\right), 5$ $\left(\hat{U}_{\infty}=13.26 \mathrm{~m} / \mathrm{s}\right)$, and $1\left(\hat{U}_{\infty}=16.29 \mathrm{~m} / \mathrm{s}\right)$. For each case, the velocities are normalized by the corresponding estimated freestream longitudinal wind speed. The amount of lidar spatial averaging in the $x, y$, and $z$ directions ranges from $\delta_{x}=2.3 \mathrm{~m}$ to $\delta_{x}=6.4 \mathrm{~m}, \delta_{y}=1.2 \mathrm{~m}$ to $\delta_{y}=5.0 \mathrm{~m}$, and $\delta_{z}=2 \mathrm{~m}$ to $\delta_{z}=3 \mathrm{~m}$, respectively. The approximate lidar probe volumes $\delta_{x} \delta_{y} \delta_{z}$ range from $8.0 \mathrm{~m}^{3}$ to $68.6 \mathrm{~m}^{3}$.

rotor disk. As expected, the velocity deficits are a strong function of the turbine's induction factor, with a maximum deficit at the rotor plane of $18 \% U_{\infty}$ occurring for case 6 , which has the highest estimated induction factor. The measurements for case 1 , with the lowest estimated induction factor, reveal velocity reductions of only $6 \% U_{\infty}$ at the rotor plane. When the turbine is stopped there is still a slight reduction in wind speed upstream of the rotor center due to the presence of the nacelle.

A comparison between the measured $U$ component velocity reductions in the induction zone and the velocity reductions predicted by the vortex sheet theory formula presented by Medici et al., and given in Eq. (4), is provided in Fig. 9. The relative velocity reductions in

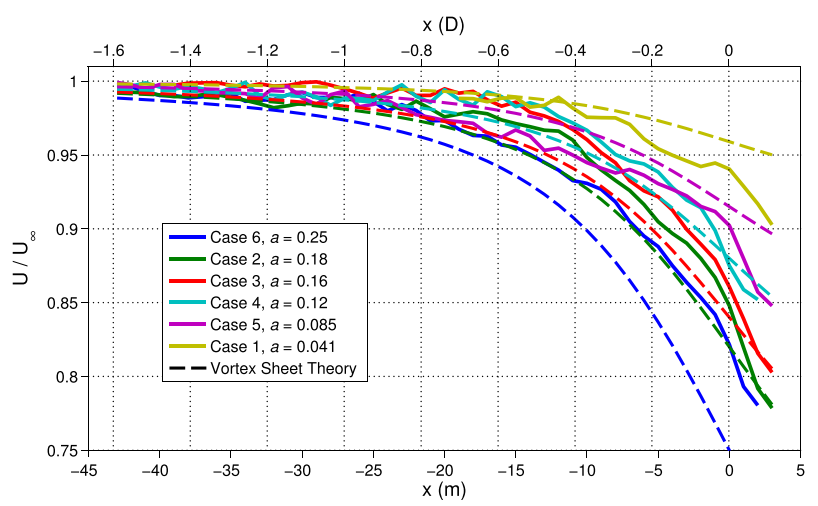

FIG. 9. Measured $U$ wind components at radial position $y=-3.375 \mathrm{~m}(-1 / 8 D)$ as a function of longitudinal position for measurement cases $6\left(\hat{U}_{\infty}=6.89 \mathrm{~m} / \mathrm{s}\right), 2\left(\hat{U}_{\infty}=8.93 \mathrm{~m} / \mathrm{s}\right), 3\left(\hat{U}_{\infty}=10.01 \mathrm{~m} / \mathrm{s}\right), 4\left(\hat{U}_{\infty}=11.49 \mathrm{~m} / \mathrm{s}\right), 5\left(\hat{U}_{\infty}=13.26 \mathrm{~m} / \mathrm{s}\right)$, and $1\left(\hat{U}_{\infty}=16.29 \mathrm{~m} / \mathrm{s}\right)$ normalized by the corresponding estimated freestream longitudinal wind speed. The measured velocities are compared with $U / U_{\infty}$ given by the vortex sheet theory formula in Eq. (4). The amount of lidar spatial averaging in the $x, y$, and $z$ directions ranges from $\delta_{x}=2.4 \mathrm{~m}$ to $\delta_{x}=5.8 \mathrm{~m}, \delta_{y}=1.3 \mathrm{~m}$ to $\delta_{y}=4.6 \mathrm{~m}$, and $\delta_{z}=2 \mathrm{~m}$ to $\delta_{z}=2.8 \mathrm{~m}$, respectively. The approximate lidar probe volumes $\delta_{x} \delta_{y} \delta_{z}$ range from $8.0 \mathrm{~m}^{3}$ to $60.7 \mathrm{~m}^{3}$. 
the induction zone for the six cases when the turbine was operating, provided in Fig. 8, are shown at the radial position $y=-3.375 \mathrm{~m}(-1 / 8 \mathrm{D})$. The corresponding vortex sheet theory estimates of the velocity reductions are formed using the estimated axial induction factors listed in Table II. Although the vortex model formula describes the velocities along the symmetry axis passing through the center of the rotor, measurements at $y=-1 / 8 D$ are shown because they include a wider range of longitudinal positions and because they are less noisy than the measurements at $y=0$ (as can be seen in Fig. 8). Although the shapes of the measured velocity reduction curves are very similar to the shape predicted by the vortex model formula, in general the measured velocity reductions are less than the theoretical reductions for high induction factors and slightly greater for low induction factors. The velocity reductions measured for cases $2,3,4$, and 6 are significantly lower than predicted by the vortex model formula. Given the very large discrepancies between the measured and predicted velocity deficits, for case 6 $(\hat{a}=0.25)$ especially, it is likely that the axial induction factors based on the V27's published power curve $\mathrm{e}^{17}$ and Eq. (2) are overestimated. This suggests that using the one-dimensional momentum theory used to derive Eq. (2) to solve for the induction factor may be an oversimplified approach. Given the extremely high values of $C_{P}$ at low wind speeds in the published power curve, however, it is also possible that the power curve is not entirely accurate. The estimated induction factor of 0.25 for case 6 corresponds to $C_{P}=0.56$, which in practice is an exceptionally high coefficient of power. For cases 1 and 5, with very low induction factors, it is likely that the presence of the tower and nacelle dominate the velocity reductions close to the rotor, causing greater velocity reductions than predicted by the vortex model, which does not include non-rotor elements. However, note that the velocity deficits in case 5, for which a MoninObukhov stability parameter of 0.047 was measured, indicative of slightly stable conditions, are significantly lower than predicted by the vortex model up to $1 \mathrm{D}$ upstream of the rotor. For cases $2,3,4$, and 6 , which contain velocity deficits that are in general lower than predicted, the calculated stability parameters indicate unstable conditions. This suggests that the atmospheric stability may impact the strength of the velocity reductions in the induction zone; unstable atmospheric conditions may reduce the velocity reductions in the induction zone while stable conditions may exaggerate the velocity deficits. But given the small amount of data obtained during stable atmospheric conditions in this campaign, further work should be performed to confirm this trend.

Similar to Fig. 8, the radial $V$ velocities for cases $1-7$, normalized by $U_{\infty}$, are plotted as a function of longitudinal position in Fig. 10 for different radial positions. Changes in the $V$ velocities due to the induction zone begin occurring between $0.5 \mathrm{D}$ and $0.75 \mathrm{D}$ upstream of the rotor and are noticeable at radial distances between $1 / 8 \mathrm{D}$ and $5 / 8 \mathrm{D}$. The maximum magnitude of the radial $V$ component occurs at the edge of the rotor, where the magnitude of $V$ for case 6 , with the highest induction factor, is $9 \% U_{\infty}$ at the rotor plane. In general, the magnitude of the $V$ component decreases for lower induction factors with only very minor radial velocities equal to $3 \% U_{\infty}$ occurring at the rotor plane for case 1, with the lowest induction factor. However, this trend is not as strong as with the $U$ velocities. For example, at a radial position of $3 / 8 D$ case 5, which has a low induction factor of 0.085 , shows $V$ magnitudes almost as high as case 6 . Because the $V$ component magnitudes are significantly smaller than the $U$ component deficits, they may be more difficult to accurately measure, however. In addition, the yaw angle of the turbine does not perfectly track the wind direction; potentially time-varying yaw error during the 10-min measurement periods could also contribute to variations in the $V$ component estimates.

By combining the $U$ and $V$ velocities measured in the horizontal scan planes, the wind direction changes in the induction zone can be determined. Figure 11 shows streamlines formed from the $U$ and $V$ velocities starting at $1 D$ upstream of the rotor for four measurement cases. As expected, the wind directions are relatively unchanged for case 7 , with the turbine stopped. The direction changes are greatest for case 6 with an estimated induction factor of 0.25 . Near the edge of the rotor the wind direction changes the most, such that a particle released $1 \mathrm{D}$ upstream of the rotor at radial position $3 / 8 D$ will, on average, end up nearly $0.04 D$ closer to the edge of the rotor when it reaches the rotor plane. For the remaining two cases, the wind 

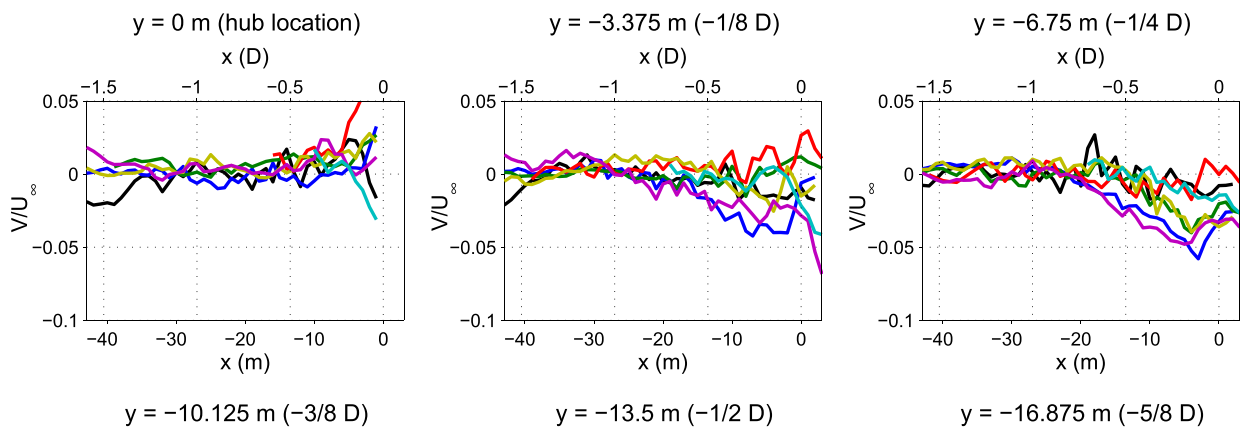

$y=-13.5 m(-1 / 2 D)$

$x(D)$
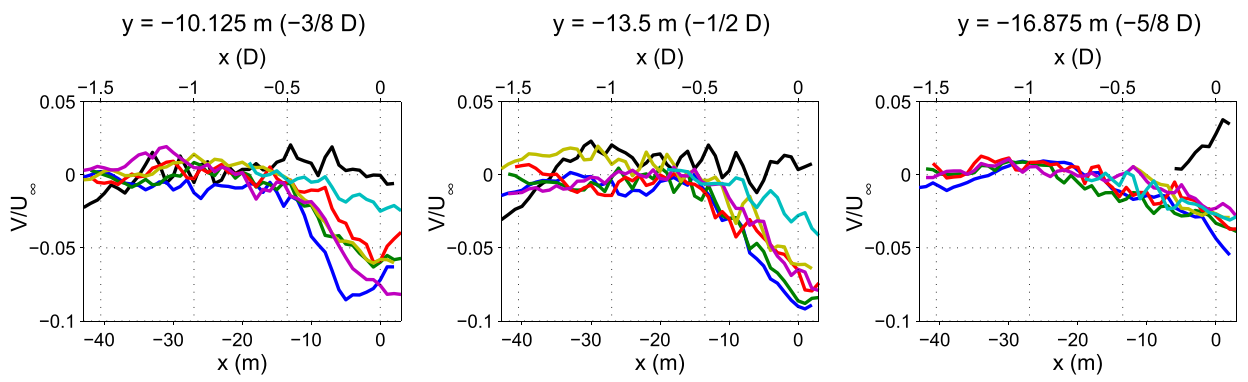

Case $7 \longrightarrow$ Case 6

$$
\text { Case } 2 \longrightarrow \text { Case } 3 \longrightarrow \text { Case } 4 \quad \text { Case } 5 \quad \text { Case } 1
$$

FIG. 10. Measured $V$ wind components as a function of radial and longitudinal position for measurement cases 7 $\left(\hat{U}_{\infty}=5.67 \mathrm{~m} / \mathrm{s}\right.$, turbine stopped $), 6\left(\hat{U}_{\infty}=6.89 \mathrm{~m} / \mathrm{s}\right), 2\left(\hat{U}_{\infty}=8.93 \mathrm{~m} / \mathrm{s}\right), 3\left(\hat{U}_{\infty}=10.01 \mathrm{~m} / \mathrm{s}\right), 4\left(\hat{U}_{\infty}=11.49 \mathrm{~m} / \mathrm{s}\right), 5$ $\left(\hat{U}_{\infty}=13.26 \mathrm{~m} / \mathrm{s}\right)$, and $1\left(\hat{U}_{\infty}=16.29 \mathrm{~m} / \mathrm{s}\right)$. For each case, the velocities are normalized by the corresponding estimated freestream longitudinal wind speed. The amount of lidar spatial averaging in the $x, y$, and $z$ directions ranges from $\delta_{x}=2.3 \mathrm{~m}$ to $\delta_{x}=6.4 \mathrm{~m}, \delta_{y}=1.2 \mathrm{~m}$ to $\delta_{y}=5.0 \mathrm{~m}$, and $\delta_{z}=2 \mathrm{~m}$ to $\delta_{z}=3 \mathrm{~m}$, respectively. The approximate lidar probe volumes $\delta_{x} \delta_{y} \delta_{z}$ range from $8.0 \mathrm{~m}^{3}$ to $68.6 \mathrm{~m}^{3}$.

direction changes along the streamlines decrease as the estimated induction factor decreases $(\hat{a}=0.18$ for case 2 and 0.12 for case 4$)$.

\section{B. Vertical scan plane}

A small amount of data was collected using the vertical plane scan pattern. Velocities calculated from a 10-min measurement period with an estimated hub-height freestream wind speed of $6.98 \mathrm{~m} / \mathrm{s}$, with a wind direction that was very close to the scan direction (case 8), are shown in Fig. 12. Fig. 12(a) shows the unnormalized velocities while Fig. 12(b) contains the velocities normalized by the estimated longitudinal freestream wind speed as a function of height,

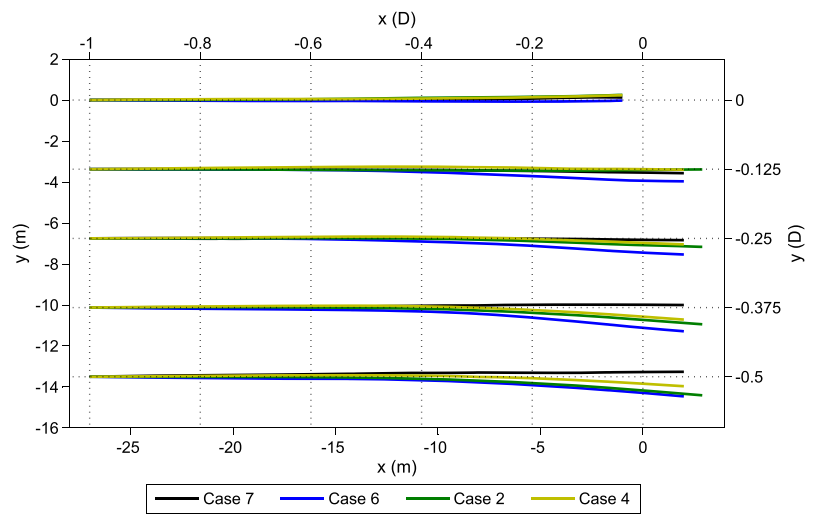

FIG. 11. Streamlines of the measured $U$ and $V$ components for measurement cases 7 ( $\hat{U}_{\infty}=5.67 \mathrm{~m} / \mathrm{s}$, turbine stopped), 6 $\left(\hat{U}_{\infty}=6.89 \mathrm{~m} / \mathrm{s}\right), 2\left(\hat{U}_{\infty}=8.93 \mathrm{~m} / \mathrm{s}\right)$, and $4\left(\hat{U}_{\infty}=11.49 \mathrm{~m} / \mathrm{s}\right)$. The amount of lidar spatial averaging in the $x, y$, and $z$ directions ranges from $\delta_{x}=2.3 \mathrm{~m}$ to $\delta_{x}=5.4 \mathrm{~m}, \delta_{y}=1.4 \mathrm{~m}$ to $\delta_{y}=3.2 \mathrm{~m}$, and $\delta_{z}=2 \mathrm{~m}$ to $\delta_{z}=2.9 \mathrm{~m}$, respectively. The approximate lidar probe volumes $\delta_{x} \delta_{y} \delta_{z}$ range from $8.0 \mathrm{~m}^{3}$ to $47.1 \mathrm{~m}^{3}$. 
(a)
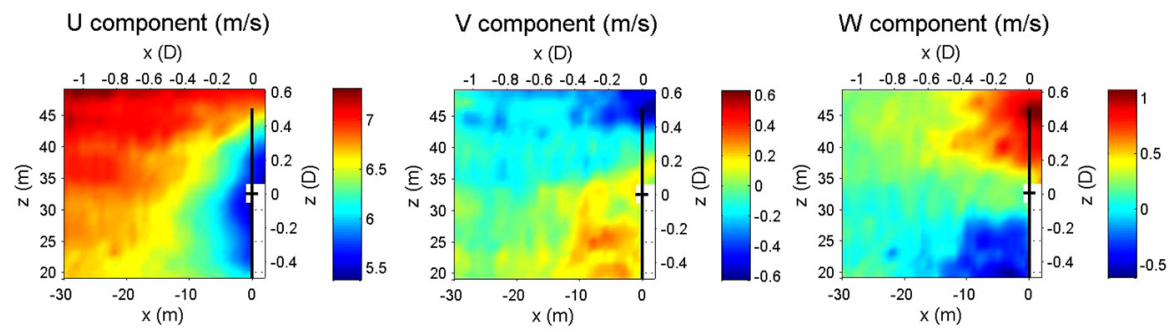

(b)
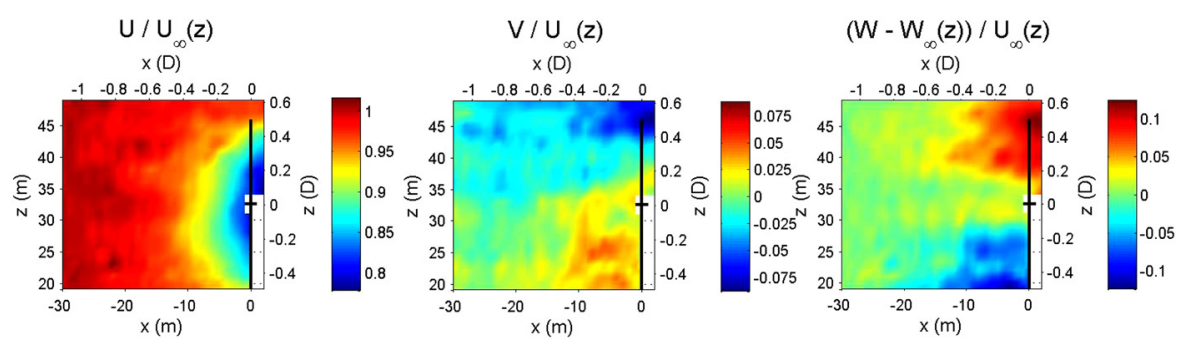

FIG. 12. Measured $U, V$, and $W$ wind components for a vertical $x z$ scan plane at the center of the rotor for measurement case $8\left(\hat{U}_{\infty}=6.98 \mathrm{~m} / \mathrm{s}\right)$ expressed as (a) unnormalized velocity components and (b) velocity components normalized by $U_{\infty}(z)$ with $W_{\infty}(z)$ subtracted from the $W$ components. The amount of lidar spatial averaging in the $x, y$, and $z$ directions ranges from $\delta_{x}=2.2 \mathrm{~m}$ to $\delta_{x}=5.5 \mathrm{~m}, \delta_{y}=2.8 \mathrm{~m}$ to $\delta_{y}=4.6 \mathrm{~m}$, and $\delta_{z}=1.2 \mathrm{~m}$ to $\delta_{z}=4.8 \mathrm{~m}$, respectively. The approximate lidar probe volumes $\delta_{x} \delta_{y} \delta_{z}$ range from $9.3 \mathrm{~m}^{3}$ to $121.3 \mathrm{~m}^{3}$.

measured 28-30 m upstream of the rotor. The vertical wind speed components plotted in Fig. 12(b) also have the estimated height-dependent mean freestream values subtracted. Velocities are only shown for $x$ distances up to $30 \mathrm{~m}$ upstream of the rotor because the wind direction of roughly $272^{\circ}$ caused lidar 2 to once again measure close to perpendicular to the wind direction for locations farther from the rotor, making the velocities at those positions difficult to estimate.

As shown in the $U$ component plots in Fig. 12, the velocity deficits in the induction zone are present upstream of the entire diameter of the rotor. The velocities at the same radial positions above and below the hub in Fig. 12(a) are different due to the wind shear present across the rotor plane extending into the induction zone. But after normalizing by the estimated mean freestream wind speed at each height, the velocity reductions appear to be symmetric about the center of the rotor. For more severe wind shear, when the induction factors at the top and bottom of the rotor differ significantly, the normalized velocity deficits present at the top and bottom of the rotor are expected to differ. Due to the slightly negative $V$ velocities above hub height and slightly positive $V$ components below hub height, it appears that there is wind veer across the rotor plane causing a slight change in wind direction with height. The source of the stronger $V$ components within $10 \mathrm{~m}$ of the rotor disk is unknown, however, but this indicates a localized exaggeration of the wind veer in the induction zone. For the vertical scan plane orientation, the $w$ component represents the radial wind speed instead of $v$, and therefore strong positive and negative vertical $W$ velocities are present near the top and bottom of the rotor disk, respectively, indicating expansion of the wind inflow around the rotor due to induced velocities produced by blade tip vortices, as was seen in the horizontal scan results. Furthermore, after subtracting the height-dependent mean freestream vertical wind speeds and normalizing by the height-dependent mean freestream longitudinal velocities, the magnitudes of the radial velocities near top and bottom edges of the rotor disk are roughly equivalent.

\section{RESULTS: TURBULENCE STATISTICS IN THE INDUCTION ZONE}

Measurement cases 9-16 are used to determine how turbulence is affected by the induction zone. Specifically, the standard deviations of the $u, v$, and $w$ wind components are calculated for different distances upstream of the rotor at different radial positions at hub height. Cases 9-12 consist of consecutive $2.5 \mathrm{~min}$ line scans performed at radial positions of $-4.5 \mathrm{~m}(1 / 6 \mathrm{D})$, 
$-9 \mathrm{~m}(1 / 3 D),-13.5 \mathrm{~m}(1 / 2 D)$, and 0 (hub location), respectively. Cases 13-16 represent the same sequence of scans but at a later time with a different wind direction and higher wind speeds. For both line scan sequences, the induction factors are relatively high, between 0.2 and 0.24 . To calculate the turbulence standard deviation values, a single $1 \mathrm{~Hz}$ time series is formed for each $1 \mathrm{~m}$-wide $x$ position bin between $x=-27 \mathrm{~m}$ ( $1 \mathrm{D}$ upstream of the rotor) and $x=0$ by interpolating between all of the lidar-measured wind speeds determined at each individual time step occurring in each respective bin. Due to the lidar scan directions intersecting with the nacelle for scan positions close to the turbine, results for scan cases 12 and 16 , with $y=0$, are only analyzed at longitudinal distances beyond $x=-3 \mathrm{~m}$.

Examples of the time series calculated from the lidar line scans in case 13 are provided in Fig. 13. The time-varying $u^{\prime}, v^{\prime}$, and $w^{\prime}$ components are plotted for five different distances upstream of the rotor at radial position $y=-4.5 \mathrm{~m}$ where zero-mean $u^{\prime}$, for example, is equal to $u-U$. Therefore, the reduction in the mean $U$ wind speed approaching the rotor is not visible (although it is shown in Fig. 15). To allow easier comparison of the turbulence at the different $x$ positions, the signals in Fig. 13 are shifted in time by time lag values that produce the highest cross correlation with the wind speeds at $x=-14 \mathrm{~m}$. In general, the large scale structures in the turbulence remain unchanged as the wind passes through the induction zone. At the rotor plane, however, small time scales of the turbulence are altered; more high frequency content is added to the turbulence. This is likely due to the altering of the wind speeds caused by velocity induction from shed blade tip vortices at the blade passage frequency of $2.15 \mathrm{~Hz}$. The turbulence in the radial $v$ component appears to change the most close to the rotor plane due to rotor induction.

Statistics for the wind speed time series calculated for cases 9-12 and 13-16 are shown in Figs. 14 and 15 , including the mean values and standard deviation values calculated at $1 \mathrm{~m}$ intervals between $x=-27 \mathrm{~m}$ and $x=0$. The mean velocity plots reveal slightly different conditions during each 2.5 min line scan for both sequences, but for each sequence the four mean wind directions deviate by less than $6^{\circ}$. The differences in mean freestream wind speed within the two scan sequences are primarily caused by the use of different averaging periods. As revealed by the presence of positive $V$ components close to the rotor in case 9 and negative $W$ components upstream of the rotor in case 10, a 2.5 min averaging period is not as capable of revealing mean wind speed trends as the 10-min averaging period used for cases $1-8$. This finding is supported by the horizontal scan plane results for different measurement periods
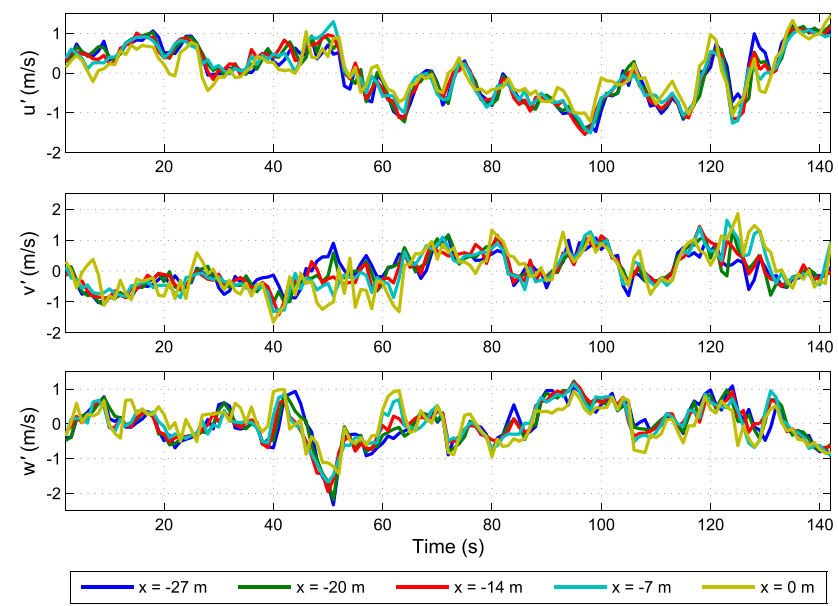

FIG. 13. Time series of measured longitudinal, transverse, and vertical wind speeds in the induction zone upstream of radial position $y=-4.5 \mathrm{~m}(-1 / 6 \mathrm{D})$ for measurement case 13 . Time series are shown for longitudinal positions $x=-27 \mathrm{~m}$ $(-1 D), x=-20 \mathrm{~m}(-0.74 D), x=-14 \mathrm{~m}(-0.52 D), x=-7 \mathrm{~m}(-0.26 D)$, and $x=0$. All time series are plotted with their mean values subtracted and are time-shifted to correct for their different positions along the mean wind direction to allow for easier comparison. The amount of lidar spatial averaging in the $x, y$, and $z$ directions ranges from $\delta_{x}=2.6 \mathrm{~m}$ to $\delta_{x}=4.5 \mathrm{~m}, \delta_{y}=3.1 \mathrm{~m}$ to $\delta_{y}=4.0 \mathrm{~m}$, and $\delta_{z}=2.2 \mathrm{~m}$ to $\delta_{z}=2.7 \mathrm{~m}$, respectively. The approximate lidar probe volumes $\delta_{x} \delta_{y} \delta_{z}$ range from $20.7 \mathrm{~m}^{3}$ to $48.4 \mathrm{~m}^{3}$. 
Mean Velocities

$x(D)$
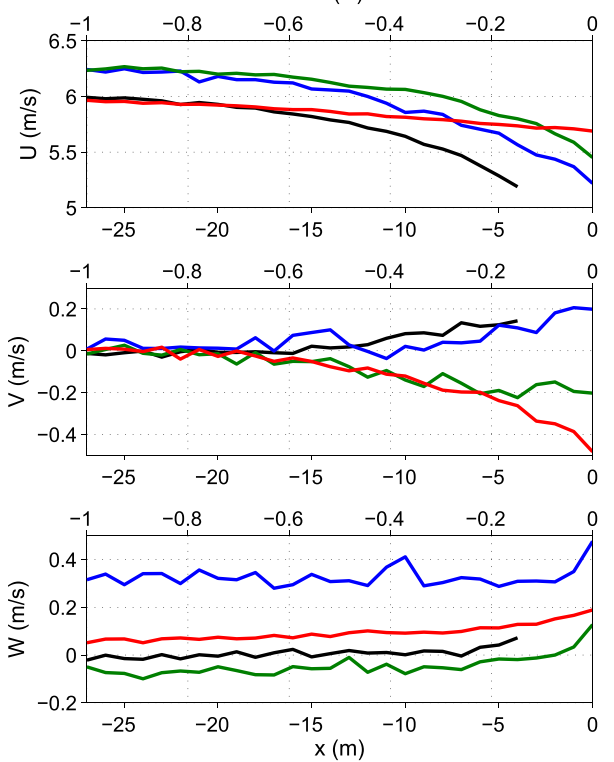

Standard Deviations

$x(D)$
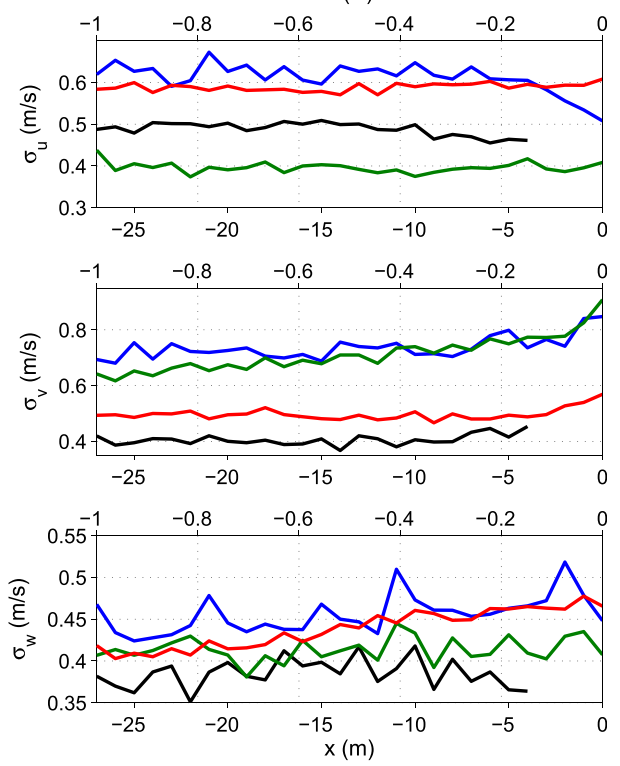

- Case $12(r=0 \mathrm{~m}) \quad$ Case $9(\mathrm{r}=-4.5 \mathrm{~m}) \quad$ Case $10(\mathrm{r}=-9 \mathrm{~m}) \quad$ Case $11(\mathrm{r}=-13.5 \mathrm{~m})$

FIG. 14. Mean and standard deviation of the measured longitudinal, transverse, and vertical wind speeds upstream of radial positions $y=0$ (hub location, case 12), $y=-4.5 \mathrm{~m}(-1 / 6 \mathrm{D}$, case 9$), y=-9 \mathrm{~m}(-1 / 3 D$, case 10$)$, and $y=-13.5 \mathrm{~m}(-1 / 2$ $D$, case 11). The amount of lidar spatial averaging in the $x, y$, and $z$ directions ranges from $\delta_{x}=2.4 \mathrm{~m}$ to $\delta_{x}=4.8 \mathrm{~m}$, $\delta_{y}=1.4 \mathrm{~m}$ to $\delta_{y}=3.1 \mathrm{~m}$, and $\delta_{z}=2.0 \mathrm{~m}$ to $\delta_{z}=2.8 \mathrm{~m}$, respectively. The approximate lidar probe volumes $\delta_{x} \delta_{y} \delta_{z}$ range from $8.4 \mathrm{~m}^{3}$ to $39.0 \mathrm{~m}^{3}$.

Mean Velocities

$x(D)$
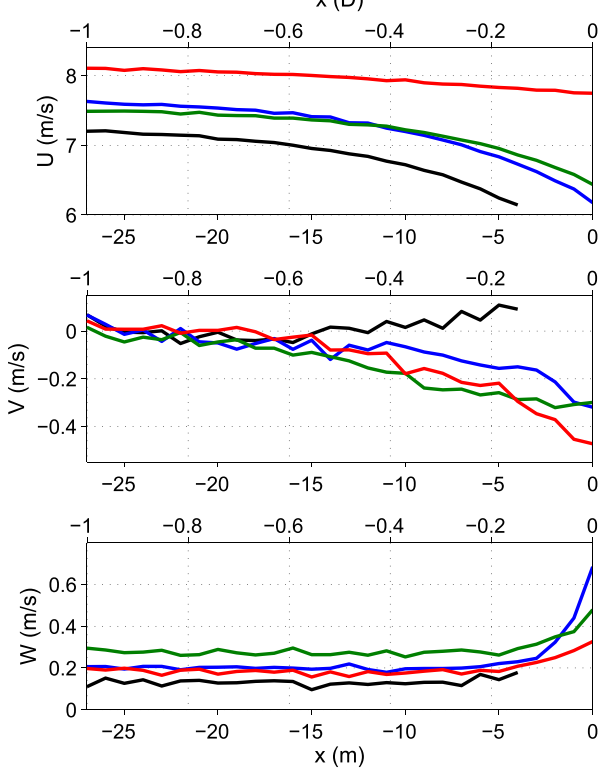

Standard Deviations
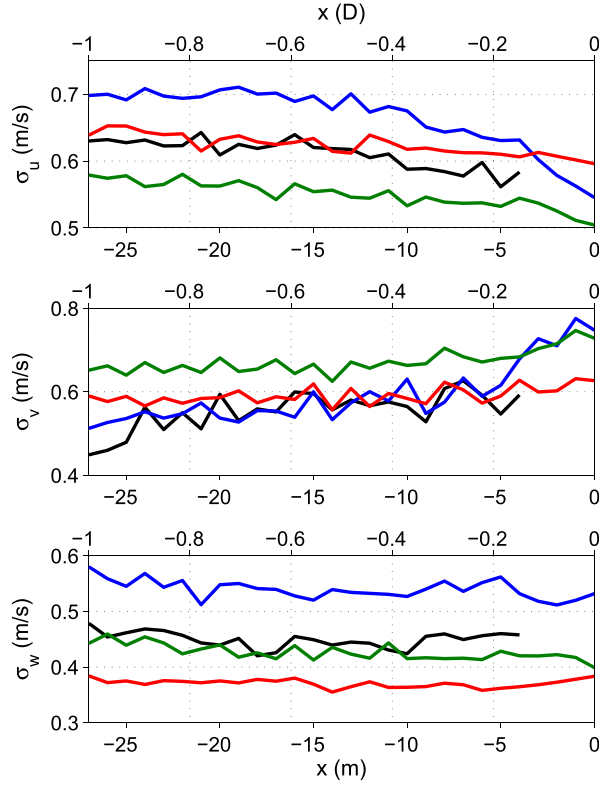

- Case $16(r=0 \mathrm{~m}) \quad$ Case $13(r=-4.5 \mathrm{~m}) \quad$ Case $14(\mathrm{r}=-9 \mathrm{~m}) \quad$ Case $15(\mathrm{r}=-13.5 \mathrm{~m})$

FIG. 15. Mean and standard deviation of the measured longitudinal, transverse, and vertical wind speeds upstream of radial positions $y=0$ (hub location, case 16$), y=-4.5 \mathrm{~m}(-1 / 6 D$, case 13$), y=-9 \mathrm{~m}(-1 / 3 D$, case 14$)$, and $y=-13.5 \mathrm{~m}(-1 / 2$ $D$, case 15). The amount of lidar spatial averaging in the $x, y$, and $z$ directions ranges from $\delta_{x}=2.6 \mathrm{~m}$ to $\delta_{x}=4.6 \mathrm{~m}$, $\delta_{y}=2.1 \mathrm{~m}$ to $\delta_{y}=4.5 \mathrm{~m}$, and $\delta_{z}=2.0 \mathrm{~m}$ to $\delta_{z}=2.8 \mathrm{~m}$, respectively. The approximate lidar probe volumes $\delta_{x} \delta_{y} \delta_{z}$ range from $12.5 \mathrm{~m}^{3}$ to $57.2 \mathrm{~m}^{3}$. 
presented in Fig. 7. The measurements in cases 9-12 in Fig. 14 show that the standard deviation of the longitudinal $u$ component is relatively unaltered as the wind travels through the induction zone, with a slight decrease close to the rotor at radial positions $y=0$ and $y=-4.5 \mathrm{~m}$. In general, the standard deviations of the radial $v$ component and vertical $w$ component, on the other hand, increase close to the rotor plane. Figure 15, which contains measurement results for cases 13-16, shows a stronger decrease in $u$ component standard deviation close to the rotor for all four radial positions investigated. Similar to cases 9-12, the standard deviation of the $v$ components also increase as the wind inflow travels toward the rotor. The $w$ component standard deviations remain relatively unchanged in the induction zone.

It is difficult to infer the exact behavior of turbulence in the induction zone from the relatively short time series in cases 9-16. However, the $u$ component standard deviations decrease slightly close to the rotor for the majority of the measurement periods. The greatest reductions occur close to the rotor center. It appears that the velocity reductions present in the mean wind speeds extend to the time-varying part of the wind inflow as well. All eight cases indicate that the radial $v$ component standard deviation increases as the inflow moves closer to the rotor, suggesting that the shedding of tip vortices by the blades creates large variations in the radial component. It is more difficult to draw conclusions about the $w$ component turbulence. Cases 9-11 reveal a slight increase in the standard deviation close to the rotor while cases 12-16 show very little variation in the induction zone. Thus the $w$ component turbulence does not appear to change significantly as the inflow approaches the rotor.

Complicating the estimates of turbulence standard deviation is the fact that the lidar spatially averages the wind speeds along the beam, as explained in Section IIE, filtering out small-scale turbulent fluctuations in the measurements. Additionally, the amount of spatial averaging as well as the beam intersection angles change depending on where in the scan pattern the lidar is measuring. Consequently, lidar range weighting could produce artificial trends in the turbulence statistics that vary depending on the distance from the turbine rotor. As discussed in Section IIE, the corner frequency of the effective low-pass filter resulting from the range weighting function with the largest FWHM during the line scans is only $\sim 0.11 \mathrm{rad} / \mathrm{m}$, which for the mean wind speed of $5.95 \mathrm{~m} / \mathrm{s}$ in case 11 is equivalent to a temporal frequency of $\sim 0.67$ $\mathrm{rad} / \mathrm{s}$ or $\sim 0.1 \mathrm{~Hz}$. Although the 3D volume averaging effect, summarized by the listed $\delta_{x}, \delta_{y}$, $\delta_{z}$, and $\delta_{x} \delta_{y} \delta_{z}$ parameters, cannot be described as a simple one-dimensional filter convolution, it is expected that the wind speed measurements will contain some filtering effects at frequencies as low as $0.1 \mathrm{~Hz}$. Regardless, the changing turbulence trends close to the rotor are believed to be partially caused by rotor induction. First of all, the probe volumes of lidars 1 and 2 are relatively large in this region, which should cause a more drastic reduction in the measured turbulence than in the center of the scan pattern or far from the rotor, yet the measured $v$ and $w$ component turbulence intensities close to the rotor increase rather than decrease. Moreover, the presence of greater reductions in $u$ component standard deviation close to the rotor at $y=0$ and $y=-4.5 \mathrm{~m}$, where induction effects are strongest, than near the edge of the rotor is unlikely to be caused solely by the increasing lidar probe volumes, which would affect all radial positions in a similar way.

\section{CONCLUSIONS}

In this paper, results from lidar measurements of the upstream induction zone of a $27 \mathrm{~m}$ rotor diameter Vestas V27 wind turbine were presented. In contrast to the majority of previous wind turbine induction zone studies, which have focused on finding the velocity deficits that are present far upstream $(2-3 D)$ of the turbine, this investigation focused on the region of the induction zone close to the turbine (within 1.5 rotor diameters). Whereas the previous lidarbased induction zone studies known to the authors relied only on line-of-sight velocities from nacelle lidars, this work was performed with three scanning ground-based lidars so that the three components of the wind could be detected. The experimental setup was designed to minimize instances when the lidars measure close to perpendicular to the wind direction, where it is difficult to estimate the very small velocity magnitudes, as well as to minimize the lidar focus 
distances and thus the amount of volume averaging due to range weighting. To help achieve these objectives, only wind speeds upstream of one half of the rotor plane were measured. The results of the investigations showed that, as expected, the reductions in the mean longitudinal wind speed in the induction zone due to rotor induction increase as the turbine's axial induction factor increases. The radial component of wind becomes non-zero near the edge of the rotor due to the expected expansion of the wind inflow around the rotor disk caused by induction from blade tip vortices. The relative magnitude of the radial component increases as the axial induction factor increases as well. No significant change in the mean tangential wind component (equivalent to the vertical component for the horizontal plane scan at hub height) was detected upstream of the turbine. Measurements of the wind speed time series upstream of the turbine reveal a general decrease in the longitudinal component turbulence standard deviation close to the rotor and an increase in the radial component standard deviation, while the tangential component standard deviation is relatively unchanged. Very close to the rotor plane, high frequency turbulence components are added to the wind speeds, likely caused by periodic induction effects from tip vortices being shed by the passing blades.

This research highlights the challenges that are associated with scanning lidar measurements. While the underlying pointing direction, focus distance, and synchronization accuracy of the lidars are very high, the measurement error due to the volume averaging inherent in the three-beam lidar measurement process is difficult to analyze. It is expected that range weighting creates a small location-dependent bias in the mean velocity measurements in the induction zone, due to the nonlinear distribution of the velocity deficits upstream of the turbine, but further work is required to estimate exactly how much error this bias causes. Additionally, the variable effective averaging volume of the lidar measurements throughout the scan pattern creates some uncertainty in determining how the turbulence statistics depend on the specific location in the induction zone. Separating the spatially varying filtering effect from the true spatial dependence of the turbulence parameters is not straightforward, and only the behavior of the low frequency turbulence, which is largely unaffected by volume averaging, can be accurately compared throughout the scan domain. Computations of the estimated transfer function of the volume averaging effect using the approach outlined by Mann et al. ${ }^{11}$ could be performed to predict the exact impact on measurement error. However, as explained in Section V, the identified turbulence trends resulting from the line scans in the induction zone are believed to be at least partially caused by rotor induction.

The conclusions drawn in this study relied on measurements upstream of half of the rotor and it was assumed that velocity reductions and radial wind speeds are similar on the other half of the rotor. Future investigations including measurements across the entire rotor disk would be useful to verify this assumption. By comparing the measured velocity reductions in the induction zone with the reductions predicted by vortex sheet theory, it was found that in general the measured velocity deficits are lower than the theoretical reductions for unstable conditions. While some of the disagreement is likely due to inaccuracies in estimating the axial induction factor of the turbine, it was also found that for the single case with slightly stable atmospheric conditions, the velocity deficits are greater than predicted. Additional measurements of the induction zone during stable conditions would be useful to determine whether this trend holds in general. Additionally, measurements performed during wind conditions with a wider variety of wind shear intensities would be useful to determine any possible correlation between the amount of wind shear and the behavior of the induction zone. Finally, future investigations using computational fluid dynamics to simulate the induction zone of the V27 turbine would be of interest for comparison with the lidar measurements presented in this work.

\section{ACKNOWLEDGMENTS}

The authors would like to acknowledge Per Hansen, Claus Brian Munk Pedersen, and Kasper Hjorth Hansen from the Department of Wind Energy at the Technical University of Denmark for their invaluable assistance deploying, operating, and troubleshooting the lidar systems during the measurement campaign. The authors gratefully acknowledge the financial support from the Danish 
Agency for Science, Technology and Innovation through Grant No. 2136-08-0022 for the support to the Danish research infrastructure facility WindScanner.dk (http://www.windscanner.dk). Support from the European Commission's FP7.WindScanner.eu Preparatory Phase project WINDSCANNER 312372 is also acknowledged. Further support for this work was provided by the U.S. National Renewable Energy Laboratory as well as by a Fulbright U.S. Student Program fellowship administered through the Danish-American Fulbright Commission.

${ }^{1}$ J. F. Manwell, J. G. McGowan, and A. L. Rogers, Wind Energy Explained: Theory, Design and Application, 2nd ed. (John Wiley \& Sons, Chichester, 2009).

${ }^{2}$ A. Segalini and P. H. Alfredsson, "A simplified vortex model of propeller and wind-turbine wakes," J. Fluid Mech. 725, 91-116 (2013).

${ }^{3}$ S. Sarmast, A. Segalini, R. F. Mikkelsen, and S. Ivanell, "Comparison of the near-wake between actuator line simulations and a simplified vortex model of a horizontal-axis wind turbine," Wind Energy (published online 2015).

${ }^{4}$ D. Medici, S. Ivanell, J. A. Dahlberg, and P. H. Alfredsson, "The upstream flow of a wind turbine: Blockage effect," Wind Energy 14, 691-697 (2011).

${ }^{5}$ E. Simley, L. Y. Pao, P. Gebraad, and M. Churchfleld, "Investigation of the impact of the upstream induction zone on LIDAR measurement accuracy for wind turbine control applications using large-eddy simulation," J. Phys.: Conf. Ser. 524, 012003 (2014).

${ }^{6}$ A. Tindal, C. Johnson, M. LeBlanc, K. Harman, E. Rareshide, and A. Graves, "Site-specific adjustments to wind turbine power curves," paper presented at the American Wind Energy Association WINDPOWER Conference, Houston, TX, 2008.

${ }^{7}$ IEC 61400-12-1, Power Performance Measurements of Electricity Producing Wind Turbines, 1st ed. (International Electrotechnical Commission, 2005).

${ }^{8}$ K. Modarresi and R. H. Kirchhoff, "The flow field upstream of a horizontal axis wind turbine," Wind Energy Center Reports, Paper 10, University of Massachusetts Wind Energy Center, Amherst, MA, 1979.

${ }^{9}$ C. Slinger, M. Leak, M. Pitter, and M. Harris, "Relative power curve measurements using turbine mounted, continuouswave lidar," in Proceedings of the European Wind Energy Association 2013 Annual Event, Vienna, Austria, 2013.

${ }^{10}$ M. Asimakopoulos, P. Clive, G. More, and R. Boddington, "Offshore compression zone measurement and visualization," paper presented at the European Wind Energy Association 2014 Annual Event, Barcelona, Spain, 2014.

${ }^{11}$ J. Mann, J.-P. Cariou, M. S. Courtney, R. Parmentier, T. Mikkelsen, R. Wagner, P. Lindelöw, M. Sjöholm, and K. Enevoldsen, "Comparison of 3D turbulence measurements using three staring wind lidars and a sonic anemometer," Meteorol. Z. 18, 135-140 (2009).

${ }^{12}$ F. Carbajo Fuertes, G. V. Iungo, and F. Portè-Agel, "3D turbulence measurements using three synchronous wind lidars: Validation against sonic anemometry,” J. Atmos. Oceanic Technol. 31, 1549-1556 (2014).

${ }^{13} \mathrm{G}$. V. Iungo and F. Portè-Agel, "Field measurements of wind turbine wakes with lidars," J. Atmos. Oceanic Technol. 30, 274-287 (2013).

${ }^{14}$ M. Sjöholm, N. Angelou, P. Hansen, K. H. Hansen, T. Mikkelsen, H. Steinar, J. A. Silgjerd, and N. Starsmore, "Twodimensional rotorcraft downwash flow field measurements by lidar-based wind scanners with agile beam steering," J. Atmos. Oceanic Technol. 31, 930-937 (2014).

${ }^{15} \mathrm{~N}$. Vasiljevic, "A time-space synchronization of coherent Doppler scanning lidars for 3D measurements of wind fields," Ph.D. dissertation (Technical University of Denmark, Roskilde, Denmark, 2014).

${ }^{16}$ Vestas V27-225 kW, $50 \mathrm{~Hz}$ wind turbine with tubular/lattice tower-general specification, Item no. 941129, version 1.2.0.24, Vestas, 1994.

${ }^{17}$ S. M. Petersen, "Wind turbine test-Vestas V27-225 kW," Technical Report No. Risø-M-2861, Risø National Laboratory, Denmark, 1990.

${ }^{18}$ T. Mikkelsen, J. Mann, M. M. Courtney, and M. Sjöholm, "Windscanner: 3-D wind and turbulence measurements from three steerable Doppler lidars,” IOP Conf. Ser.: Earth Environ. Sci. 1, 012018 (2008).

${ }^{19}$ N. Angelou, J. Mann, M. Sjöholm, and M. Courtney, "Direct measurement of the spectral transfer function of a laser based anemometer," Rev. Sci. Instrum. 83, 033111 (2012).

${ }^{20}$ N. Angelou, F. F. Abari, J. Mann, T. Mikkelsen, and M. Sjöholm, "Challenges in noise removal from Doppler spectra acquired by a continuous-wave lidar," in Proceedings of the 26th International Laser Radar Conference, Porto Heli, Greece, 2012.

${ }^{21}$ P. J. M. Clive, "Compensation of vector and volume averaging bias in lidar wind speed measurements," IOP Conf. Ser.: Earth Environ. Sci. 1, 012036 (2008).

${ }^{22}$ E. Simley, L. Y. Pao, R. Frehlich, B. Jonkman, and N. Kelley, "Analysis of light detection and ranging wind speed measurements for wind turbine control," Wind Energy 17, 413-433 (2014).

${ }^{23}$ J. C. Wyngaard, Turbulence in the Atmosphere (Cambridge University Press, Cambridge, 2010). 